\title{
Generation of a Synthetic Human Chromosome with Two Centromeric Domains for Advanced Epigenetic Engineering Studies
}

Elisa Pesenti, ${ }^{\dagger}$ Natalay Kouprina, ${ }^{\ddagger}$ Mikhail Liskovykh, ${ }^{\ddagger}$ Joan Aurich-Costa, ${ }^{\S}$ Vladimir Larionov, ${ }^{\ddagger}$ Hiroshi Masumoto," William C. Earnshaw, ${ }^{* \dagger}{ }^{\dagger}$ and Oscar Molina*, ${ }^{*}, \perp \odot$

${ }^{\dagger}$ Wellcome Trust Centre for Cell Biology, University of Edinburgh, Edinburgh EH9 3QR, United Kingdom

${ }^{\ddagger}$ Genome Structure and Function Group, Developmental Therapeutics Branch, National Cancer Institute, National Institutes of Health, Bethesda, Maryland 20892, United States

${ }^{\S}$ Research and Development, Cellay Inc., Cambridge, Massachusetts 02139, United States

"Laboratory of Cell Engineering, Department of Frontier Research, Kazusa DNA Research Institute, Kisazaru 292-0818, Japan

${ }^{\perp}$ Josep Carreras Leukaemia Research Institute, School of Medicine, University of Barcelona, Casanova 143, 08036 Barcelona, Spain

\section{Supporting Information}

ABSTRACT: It is generally accepted that chromatin containing the histone $\mathrm{H} 3$ variant CENP-A is an epigenetic mark maintaining centromere identity. However, the pathways leading to the formation and maintenance of centromere chromatin remain poorly characterized due to difficulties of analysis of centromeric repeats in native chromosomes. To address this problem, in our previous studies we generated a human artificial chromosome (HAC) whose centromere contains a synthetic alpha-satellite (alphoid) DNA array containing the tetracycline operator, the alphoid ${ }^{\text {tetO }}-$ HAC. The pres-

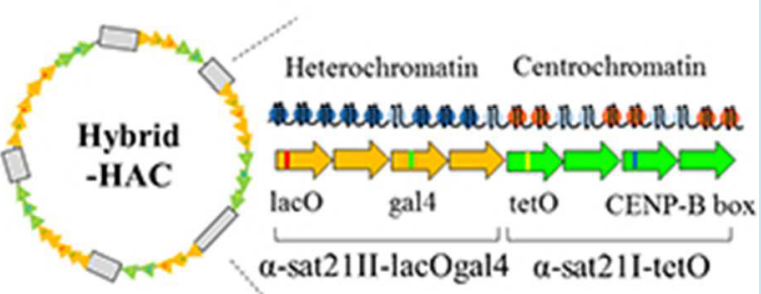
ence of tetO sequences allows the specific targeting of the centromeric region in the HAC with different chromatin modifiers fused to the tetracycline repressor. The alphoid ${ }^{\text {tetO }}-\mathrm{HAC}_{\mathrm{H}}$ has been extensively used to investigate protein interactions within the kinetochore and to define the epigenetic signature of centromeric chromatin to maintain a functional kinetochore. In this study, we developed a novel synthetic HAC containing two alphoid DNA arrays with different targeting sequences, tetO, lacO and gal4, the alphoid ${ }^{\text {hybrid }}$-HAC. This new HAC can be used for detailed epigenetic engineering studies because its kinetochore can be simultaneously or independently targeted by different chromatin modifiers and other fusion proteins.

KEYWORDS: centromere, chromosome segregation, human artificial chromosome, kinetochore, mitosis

$\mathrm{C}$ entromeres define the site of the assembly of the kinetochore, a multiprotein complex that directs chromosome segregation during cell division..$^{1}$ In humans, endogenous centromeres typically form on chromosome-specific higherorder alphoid DNA arrays, which are composed of $171 \mathrm{bp}$ alphasatellite monomer units that are tandemly arranged in a directional head-to-tail fashion. ${ }^{2}$ Independent of this sequence preference, specific deposition of the centromere-specific histone $\mathrm{H} 3$ variant CENP-A forms the basis for an epigenetic maintenance of centromere identity. ${ }^{3,4}$ The epigenetic control of centromere activity is illustrated by the inactivation of centromeres on dicentric chromosomes 5 and by the formation of rare neocentromeres that recruit CENP-A and assemble fully functional kinetochore structures on nonalphoid DNA. ${ }^{6}$

Microscopic investigation of stretched kinetochore fibers revealed that blocks of CENP-A nucleosomes are interspersed with canonical histone $\mathrm{H} 3$ nucleosomes that contain transcriptionassociated modifications, such as $\mathrm{H} 3 \mathrm{~K} 4 \mathrm{me} 2$ and $\mathrm{H} 3 \mathrm{~K} 36$ me2. ${ }^{7-9}$ This special chromatin was termed "centrochromatin" 7 and suggests a functional link between the local chromatin environment and kinetochore function. ${ }^{1}$ These observations raise several questions about the exact nature of the chromatin that specifies kinetochore assembly and propagation. (1) What combination of histone modifications defines the elusive epigenetic state that is centrochromatin? (2) Can histone modifications be manipulated to turn normal chromatin into centrochromatin or, inversely, to inactivate established centromeres? (3) What barriers prevent heterochromatin spreading into centromeres? Answering these questions with native human chromosomes is extremely challenging.

Knowledge of the structure and function of human centromeres has dramatically increased since the first reconstitution of functional human centromeres, when DNA satellite repeats were transfected into human cells, forming the first human artificial chromosomes (HACs). ${ }^{10,11}$ Subsequently, many HACs have been constructed using different strategies of satellite DNA cloning and delivery into human cells ${ }^{12-23}$

The development of RCA-TAR cloning by our group provided the first method for constructing synthetic alphoid DNA arrays

Received: January 11, 2018

Published: March 22, 2018 


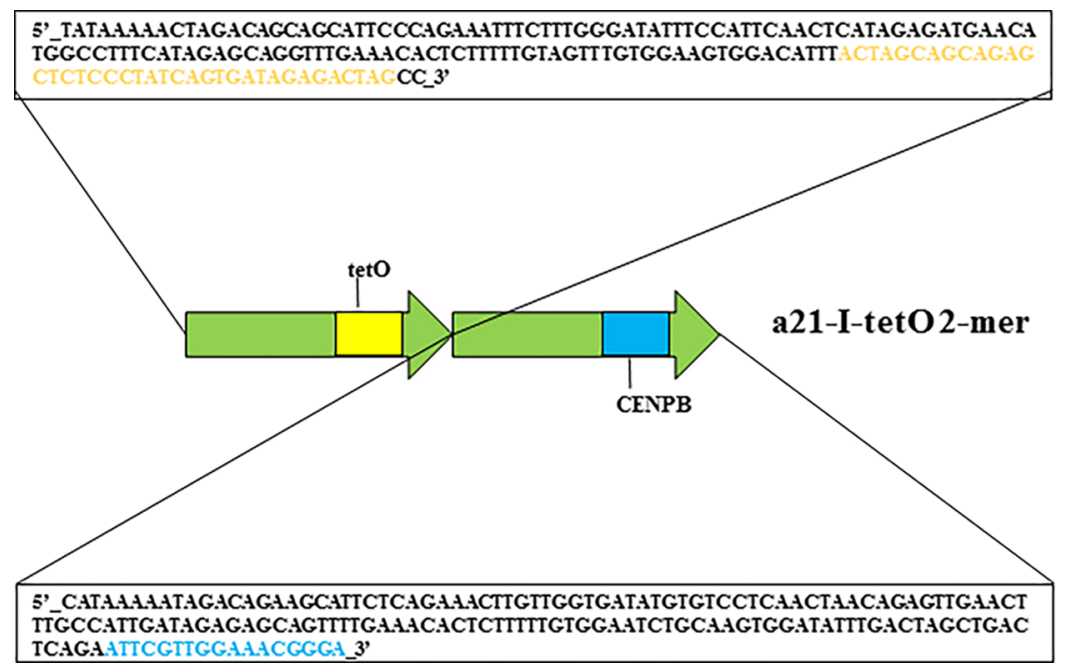

Figure 1. Sequence of the alphoid dimer used for construction of the $\alpha 21$-I-tetO array. Both monomers are derived from a chromosome 21 alphoid type I (HOR). One monomer contains a CENP-B box (shaded blue). In the second monomer, the position corresponding to the CENP-B box sequence was replaced by a $42 \mathrm{bp}$ tetO motif (shaded yellow).

with precisely defined DNA sequence variation and the possibility of manipulating alphoid DNA arrays. ${ }^{24}$ This approach involves rolling circle amplification (RCA) of alphoid DNA oligomers as small as a dimer followed by assembly of the amplified fragments by transformation-associated recombination (TAR) in yeast. ${ }^{25,26}$ Using the RCA-TAR method, synthetic alphoid DNA arrays up to $140 \mathrm{~kb}$ have been generated and used for de novo HAC formation. ${ }^{24}$ The method also permitted generation of a synthetic HAC with a conditional centromere, the alphoid ${ }^{\text {tetO }}-\mathrm{HAC}^{27}$ which has been instrumental in resolving a role for chromatin structure in kinetochore function. ${ }^{23}$ The alphoid $^{\text {tetO }}$-HAC is based on a dimeric alphoid DNA array that contains alternating monomers with either CENP-B boxes or tetracycline operator (tetO) sequences. The latter sequences allow specific engineering of the alphoid ${ }^{\text {tetO }}-\mathrm{HAC}$ using chromatin modifiers fused to the tetracycline repressor (tetR). The alphoid ${ }^{\text {tetO }}$-HAC has been extensively used to investigate protein interactions within the kinetochore and to define the epigenetic signature of centromeric chromatin to maintain a functional kinetochore. ${ }^{9,23,28-33}$ Studies with this HAC revealed that nucleating heterochromatin or high levels of active transcription within centrochromatin disrupts kinetochore function ${ }^{27-29}$ and that a low level of transcription is needed to maintain an active kinetochore. ${ }^{9,33}$ Recent studies using the alphoid ${ }^{\text {tetO }}-\mathrm{HAC}$ showed that histone modifications and centromeric transcription block heterochromatin spreading into centrochromatin, thus preventing kinetochore inactivation. ${ }^{33}$ Histone modifications linked to transcription might act as an epigenetic barrier between centrochromatin and pericentromeric heterochromatin.

Although heterochromatin occurs in close proximity to centromeres, its role in chromosome segregation varies among species. S. pombe may provide an extreme example with its dependence on heterochromatin for de novo deposition of CENP-A and for proper cohesion dynamics. ${ }^{34}$ In contrast, the role of pericentromeric heterochromatin on kinetochore function in humans remains poorly understood. Results from different groups showed that disruption of pericentromeric heterochromatin is associated with chromosome mis-segregation and tumorigenesis. ${ }^{35-37}$

In this work, we have constructed a new alphoid ${ }^{\text {hybrid }}$-HAC containing two distinct synthetic alphoid arrays to investigate the role of heterochromatin domains on kinetochore maintenance and function. This HAC is based on a synthetic hybrid array consisting of an alphoid ${ }^{\text {tetO }}$ array linked to a novel alphoid DNA array derived from monomeric alpha-satellite DNA lacking CENP-B boxes and containing lac operators (lacO) and yeast Gal4 binding sites. Transfection of the hybrid array into human HT 1080 cells resulted in HAC formation. The alphoid ${ }^{\text {hybrid }}$-HAC was used to examine whether heterochromatin or centrochromatin can be induced to spread laterally by targeting chromatin modifiers.

\section{RESULTS AND DISCUSSION}

Construction of the Hybrid tetO/lacOgal4 Synthetic Arrays by RCA-TAR Cloning. We wished to construct a human artificial chromosome (HAC) containing centrochromatin and pericentromeric heterochromatin domains (alphoid ${ }^{\text {hybrid-HAC) }}$ to manipulate the epigenetic status of both domains independently. As the basis for the kinetochore domain, we designed a $343 \mathrm{bp}$ alphoid 21-I dimer from high-order repeats (HORs) of chromosome 21 in which sequences corresponding to the CENP-B box in one monomer were replaced by a 42 bp tetracycline operator sequence (tetO), the binding site for $E$. coli tetracycline repressor (tetR) (Figure 1). As the basis for the pericentromeric heterochromatin domain, we synthesized a novel 2,078 bp alphoid 21-II-lacOgal4 12-mer that lacked CENP-B boxes but contained four gal4 binding sites (each $21 \mathrm{bp}$ in size) and four lacO sequences (each $36 \mathrm{bp}$ in size). These permit targeting the heterochromatin domain with chimeric proteins containing the yeast gal4 protein and/or E. coli lactose repressor (lacI) (Figure 2).

$\mathrm{HAC}$ formation requires input naked alphoid DNA of at least $30 \mathrm{~kb}$ in size for functional CENP-A core assembly. ${ }^{38}$ For this purpose, an artificial alphoid 21-I ( $\alpha 21-\mathrm{I})$ dimer and a synthetic $\alpha 21$-II-lacOgal4 12-mer were extended by rolling circle amplification (RCA) using phage $\phi 29$ DNA polymerase followed by yeast Transformation-associated recombination (TAR) cloning (Figure 3). ${ }^{24,25}$ These steps yielded two arrays, i.e. $\sim 40 \mathrm{~kb} \alpha 21$-ItetO and $\sim 40 \mathrm{~kb} \alpha 21$-II-lacOgal4 arrays, cloned into the 10,209 bp YAC/BAC-based RCA-Sat43 vector containing the mammalian selectable marker blasticidin (Bsr) ${ }^{24}$ (Figures $3 \mathrm{~b}$, 4,a and $4 \mathrm{~b}$; see Materials and Methods for details). The tandem repeat structure of the $\alpha 21-\mathrm{II}-\mathrm{lacOgal} 4$ array was confirmed by AlwN1 


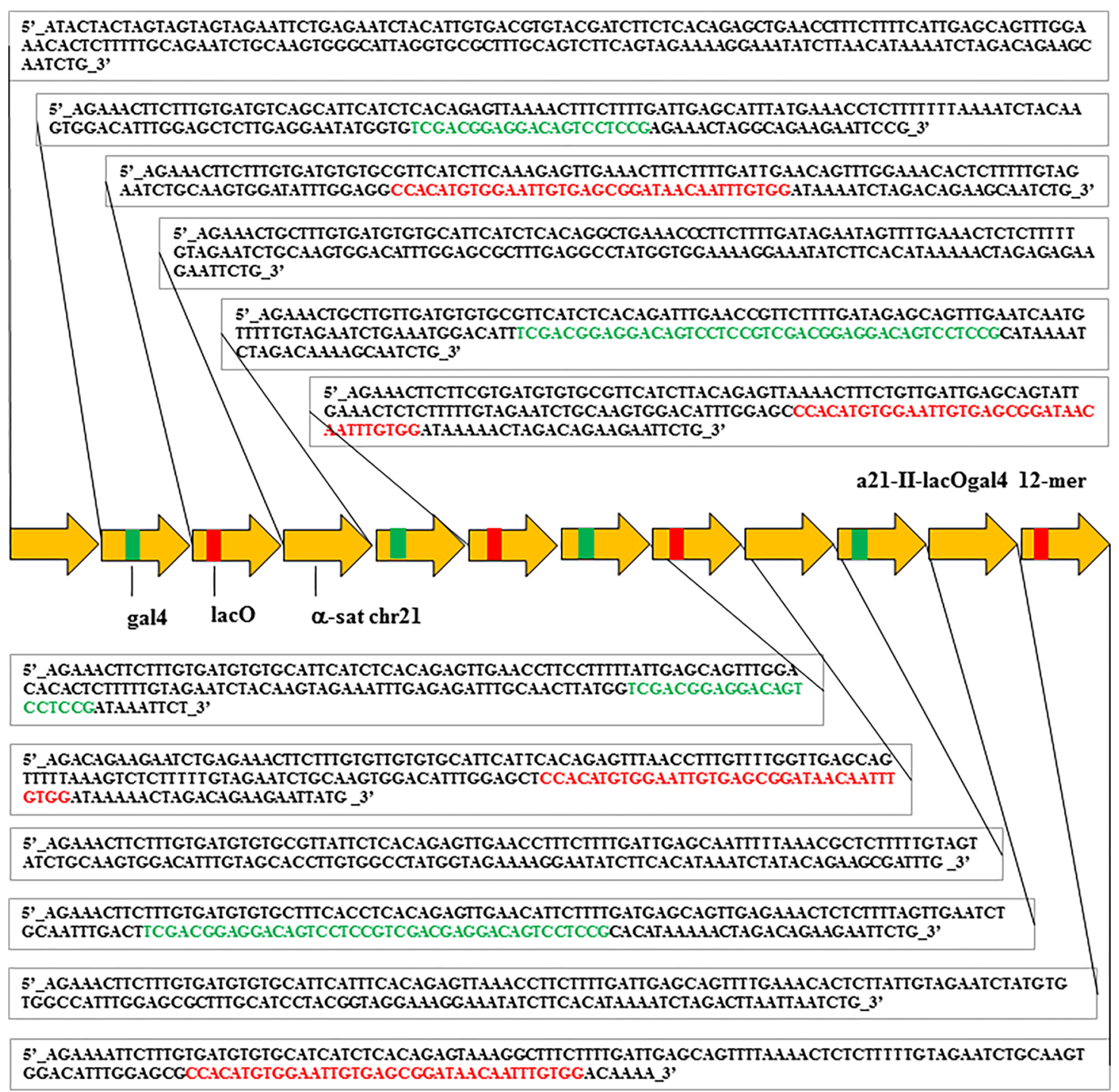

Figure 2. Sequence of the alphoid 12-mer used for construction of the $\alpha 21$-II-lacOgal4 array. All monomers were derived from a chromosome 21 alphoid type II array. Four gal4 sequences (two $21 \mathrm{bp}$ in size and two dimers $42 \mathrm{bp}$ in size; shaded green) and four lacO sequences (each $36 \mathrm{bp}$ in size; shaded red) were incorporated into a 12-mer. Fifteen MseI sites are present in the 12-mer.

digestion, which revealed a 2,078 bp 12-mer (Figure 4c). The tandem repeat structure of the $\alpha 21$-I-tetO array was confirmed by EcoRI digestion, which revealed a 343 bp 2-mer (Figure 4d).

To generate a hybrid array containing both $\alpha 21$-I-tetO and $\alpha 21$-II-lacOgal4, the two arrays were combined by recombination in yeast. After screening $\sim 190$ colonies by CHEF gel electrophoresis, we obtained a molecule consisting of $25 \mathrm{~kb} \alpha 21$ II-lacOgal4 and $30 \mathrm{~kb} \alpha 21$-I-tetO arrays in the RCA-Sat43 vector (Figures $3 \mathrm{c}$ and $4 \mathrm{e}$ ). This hybrid YAC/BAC DNA molecule of $\sim 65 \mathrm{~kb}$ in size $(\sim 55 \mathrm{~kb}$ alphoid array plus $\sim 10 \mathrm{~kb}$ vector $)$ was moved to bacterial cells and served as the input DNA for further HAC formation.

HAC Formation Using the Hybrid $\alpha$-Satellite Array in Human HT1080 Cells. For HACs to be obtained, the RCASat43 vector containing the hybrid array was purified from largescale bacterial cultures (see Materials and Methods). The quality of the hybrid array DNA was checked by CHEF gel electrophoresis (data not shown). Input DNA was transfected into human HT1080 cells, and blasticidin S (BS)-resistant clones were selected for cytogenetic analysis. A total of 17 BS-resistant clones were obtained and expanded after transfection of the hybrid array DNA construct. To determine the fate of the hybrid array in HT1080 cells (expected to be either episomal HACs or chromosome integrations), these clones were processed for fluorescence in situ hybridization (FISH) using the RCA-Sat43 vector backbone as DNA probe directly labeled with Spectrum Green (Abbott Molecular) (Figure 5a). FISH analyses showed that most of the BS-resistant clones (13 out of $17 ; 76.5 \%$ ) contain array integrations into endogenous chromosome arms (Figure $5 b$ ). Indeed, the most common fate of the input DNA construct in HAC formation experiments is integration into endogenous chromosome arms ${ }^{11,24,27,39}$ (Figure $5 b$ ). No preferential chromosomes or chromosome positions were observed for these array integrations.

FISH analysis revealed that the hybrid array formed HACs in 2 of 17 BS-resistant cell lines (clones 20.05 and 20.07), showing 11 and $60 \%$ of HAC-containing metaphases, respectively (Figure $5 \mathrm{~b}$ ). Clone 20.05 also showed metaphases containing array integrations into endogenous chromosomes. A third clone (clone 20.22) showed a single HAC-containing metaphase plus $40 \%$ of metaphases containing array integrations (Figure $5 b$ ) and was not considered as a HAC-containing cell line. 


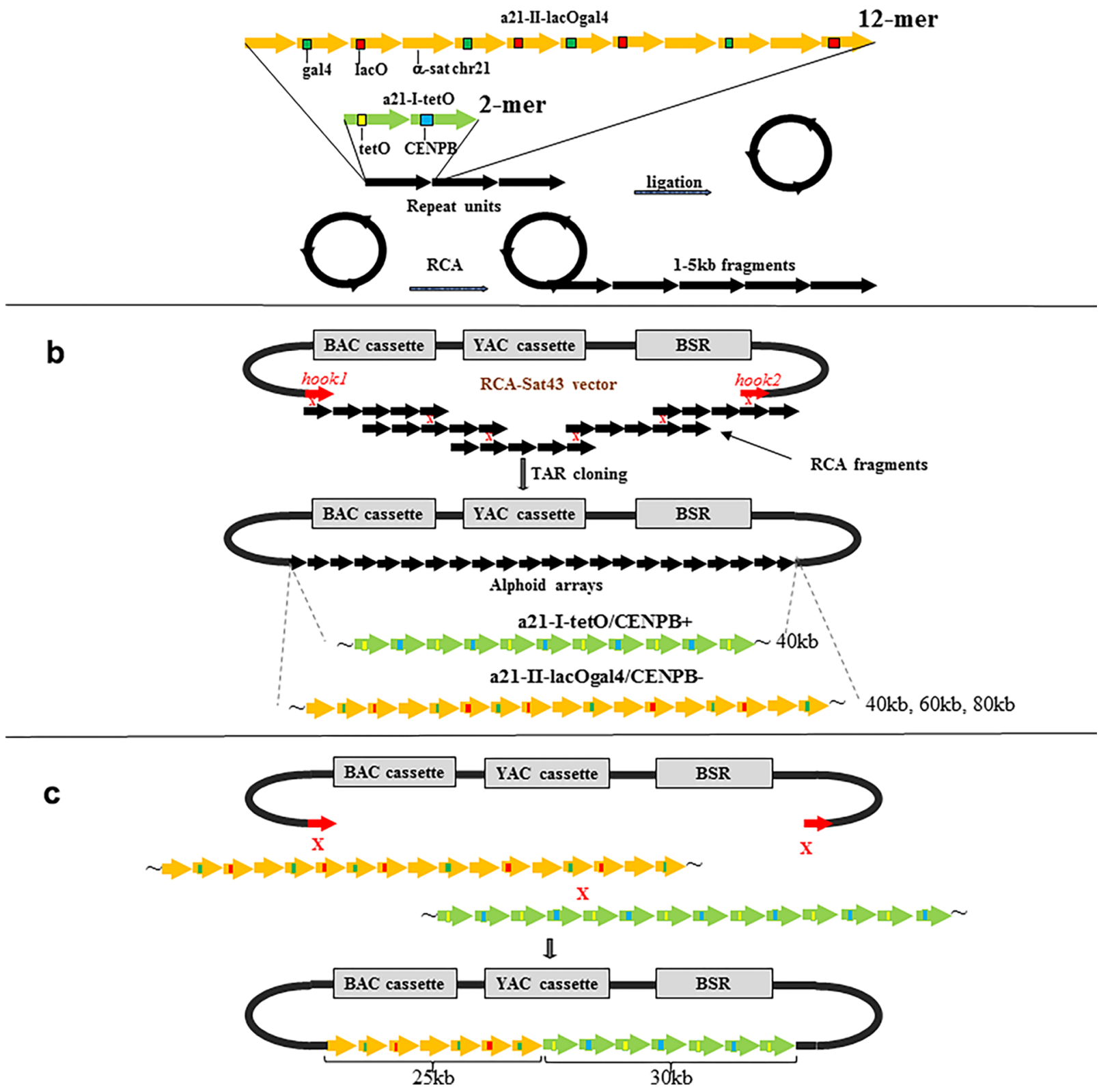

Figure 3. Schematic representation of construction of synthetic tandem arrays. (a) Step one includes amplification of either a 2,078 bp 21-II-lacOgal4 12 -mer or a 343 bp 21-I-tetO dimer by rolling circle amplification (RCA) reaction up to 1-3 kb fragments. (b) Step two includes construction of long alphoid arrays by transformation-associated recombination (TAR) cloning in yeast. The RCA-amplified fragments are cotransformed into yeast cells along with the MluI-linearized RCA-Sat43 vector (the MluI restriction site is located between the hooks). This vector contains a BAC cassette (a BAC replicon and a $\mathrm{Clm}$ marker), a YAC cassette (a selectable marker HIS3, a centromere sequence CEN6 from yeast chromosome VI, and yeast origin of replication ARSH4), and a mammalian marker Bsr (the blasticidin gene) that allows the vector to propagate in yeast, bacterial, and mammalian cells and alphoid-specific hooks of $40 \mathrm{bp}$ each (Ebersole et al. 2005). Recombination of the RCA-amplified fragments accompanied by their recombination with the hooks results in the rescue of long arrays as circular YAC/BACs, and $40 \mathrm{~kb} \alpha 21$-I-tetO and $40 \mathrm{~kb} \alpha 21$-II-lacOgal4 arrays were chosen for further experiments. (c) Construction of the hybrid tetO-CENPB ${ }^{+}$-lacOgal4-CENPB ${ }^{-}$array. Recombination between the arrays accompanied by their recombination with the vector hooks leads to formation of the hybrid arrays. Ultimately, a molecule containing a $20 \mathrm{~kb}$ lacOgal4 array and $25 \mathrm{~kb}$ tetO array was chosen for HAC formation.

Only clones showing a minimum of $5 \%$ of metaphases with detectable HACs (at least 2 out of the 25 metaphases analyzed) were considered. The remaining BS-resistant clones showed neither HACs nor array integrations in any metaphase analyzed, suggesting that the clones may have acquired only the BSR gene. Overall, we observed HAC formation with an efficiency of $11.7 \%$ after transfection of the hybrid alphoid DNA array. This is consistent with the efficiency observed previously by others using synthetic alphoid DNA arrays for HAC formation experiments. ${ }^{24,27}$

These results showed that the hybrid array can form HACs in human HT1080 cells with an efficiency similar to those of other synthetic alphoid DNA arrays. 


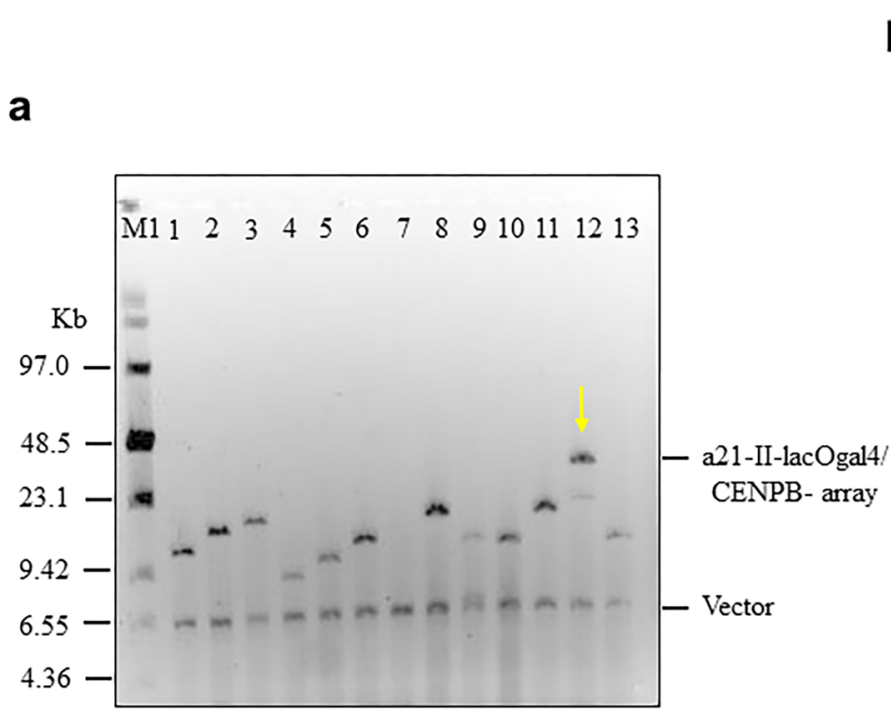

b
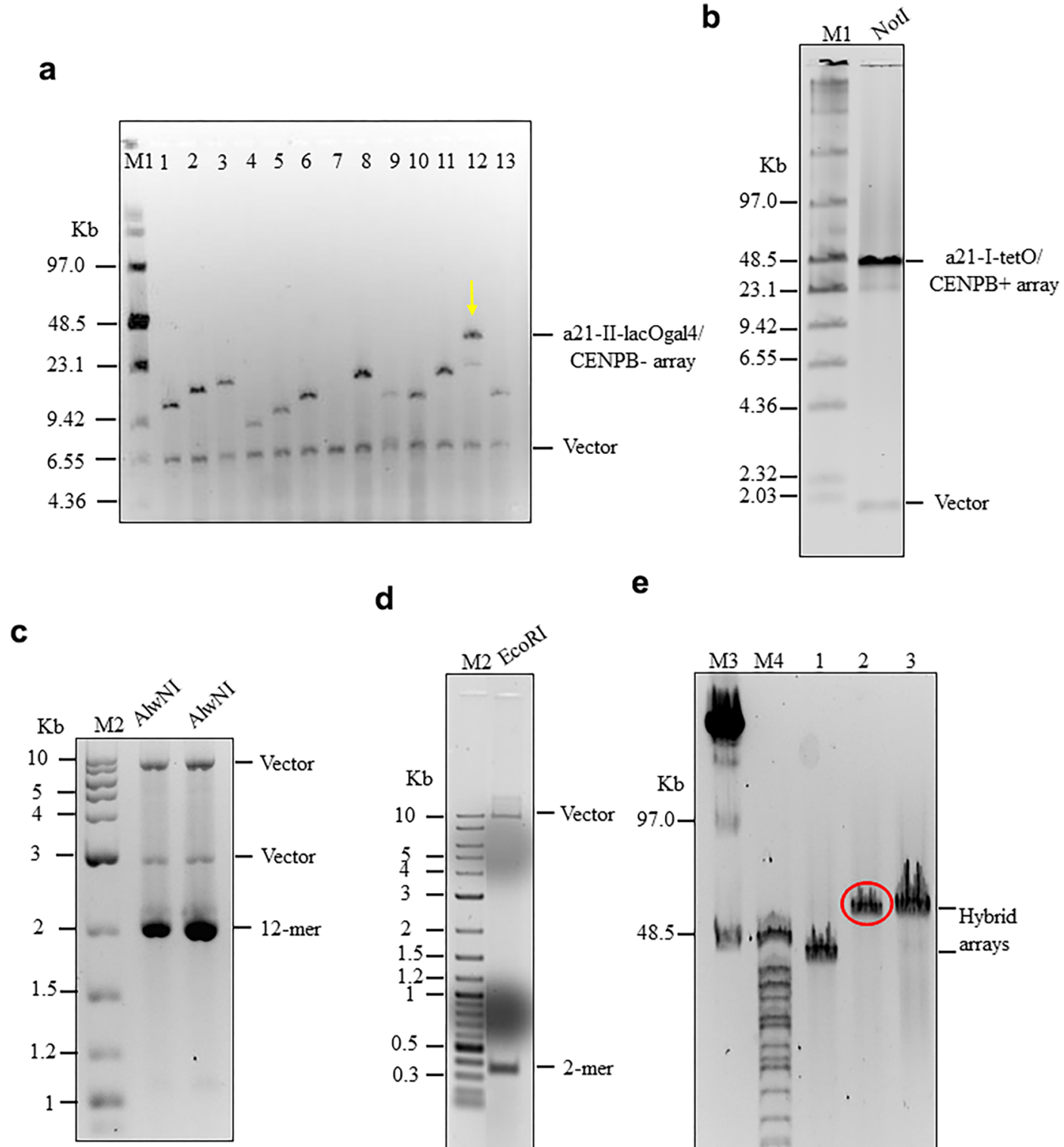

d
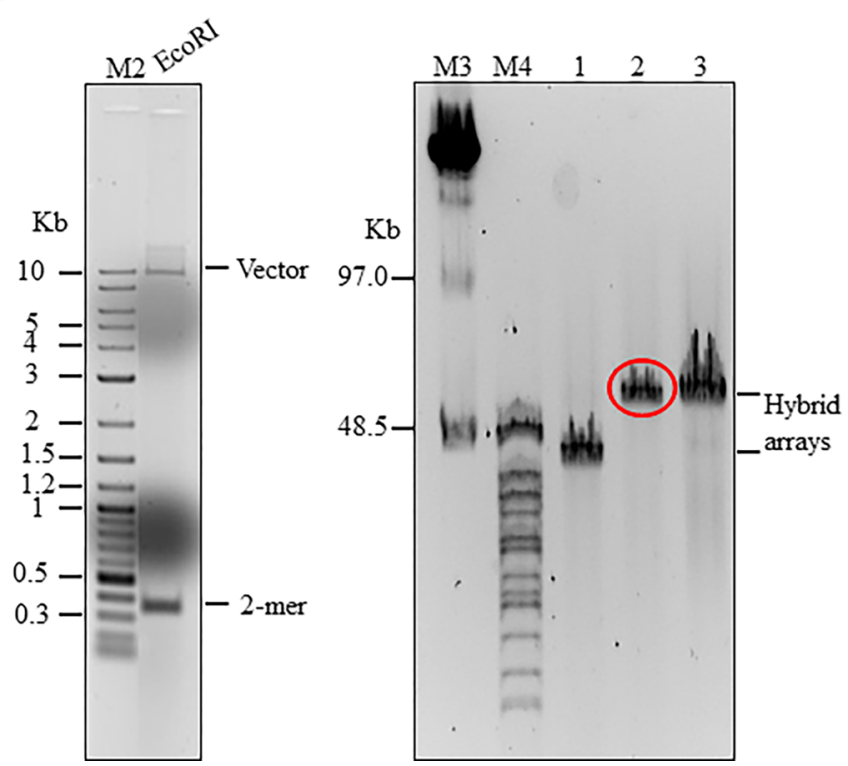

Figure 4. Hybrid alphoid-DNA array construction. (a) CHEF analysis of 13 BACs with $\alpha 21$-II-lacOgal4 arrays of different size. The BAC DNAs were linearized by AvaII to release a vector part and an array. BAC \#12 has an array of $\sim 40 \mathrm{~kb}$ in size. (b) CHEF analysis of the BAC with the $\alpha 21$-I-tetO array of $\sim 40 \mathrm{~kb}$ in size. The BAC DNA was digested by NheI/SpeI to release a vector fragment and the array. (c) Confirmation of the tandem repeat structure of 40 and $60 \mathrm{~kb} \alpha 21$-II-lacOgal4 arrays by AlwN1 digestion. CHEF analysis revealed 2,461 bp 12-mer $\alpha 21$-II-lacOgal4 repeat units. (d) Conformation of the tandem repeat structure of a $40 \mathrm{~kb} \alpha 21$-I-tetO array by EcoRI digestion. CHEF analysis revealed $343 \mathrm{bp} 2$-mer 21-I-tetO repeat units. (E) CHEF analysis of the hybrid $\alpha 21$-I-tetO/a21-II-lacOgal4 arrays. (Lane 1) Array consisting of $10 \mathrm{~kb}$ of the $\alpha 21$-II-lacOgal4 array and $30 \mathrm{~kb}$ of the a21-I-tetO array. (Lane 2) Array consisting of $25 \mathrm{~kb} \alpha 21$-II-lacOgal4 array and $30 \mathrm{~kb} \alpha 21$-I-tetO array. The array in lane 2 (in red) was chosen for HAC formation. (Lane 3) Array consisting of $15 \mathrm{~kb} \alpha 21$-II-lacOgal4 array and $40 \mathrm{~kb} \alpha 21$-I-tetO array.

CENP-A Overexpression Significantly Increases the Efficiency of HAC Formation. The low efficiency of de novo HAC formation is one limitation for the wider application of HAC technology. ${ }^{20,23,40}$ We were therefore interested when it was recently reported that elevated levels of CENP-A increase the frequency of centrochromatin assembly and minichromosome formation in S. pombe. ${ }^{41}$ Moreover, we previously observed that seeding of centromeric chromatin at ectopic array integrations under different conditions is enhanced in cells overexpressing CENP-A. ${ }^{42}$

To investigate the effect of CENP-A levels on the efficiency of de novo HAC formation, we transfected the hybrid array into HT1080 cells transiently overexpressing CENP-A. As in previous
HAC formation experiments, BS-resistant clones were selected and further expanded for cytogenetic analysis. FISH experiments revealed HACs in 11 of the 23 BS-resistant clones obtained after transfection with a range of $8-76 \%$ of HAC-containing metaphases (Figure 5c). Two additional clones showed HACs in a single metaphase and therefore were not considered as HAC-positive cell lines (Figure 5c). Some clones showed both array integrations and HACs as previously described for other HACs. ${ }^{27,43}$ For further analysis, we chose two clones, 20.CA.07 and 20.CA24 (Figure 5c), that contained only autonomously replicating HACs.

Thus, transient CENP-A overexpression increases the efficiency of HAC formation by 3-fold (Figure 5d), overcoming an important limitation of the HAC technology. 


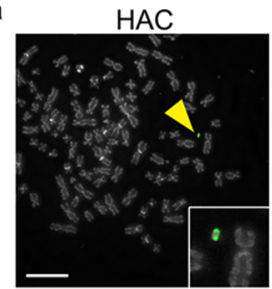

CENP-A OVEREXPRESSION
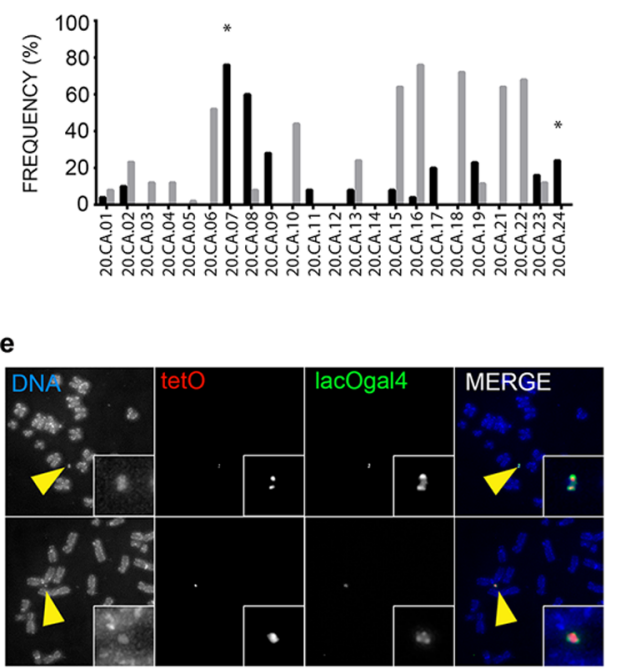

h

HAC STABILITY

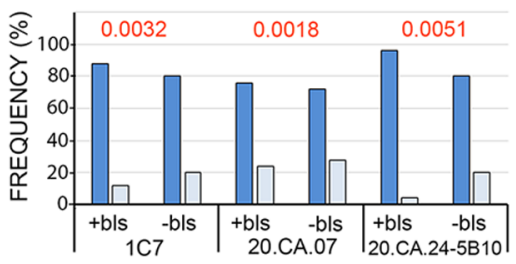

$1 \mathrm{HAC} \quad \square 0 \mathrm{HAC}$ b

INTEGRATION

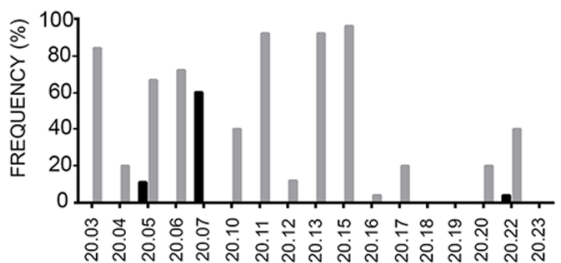

HAC FORMATION

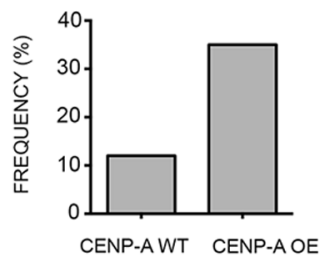

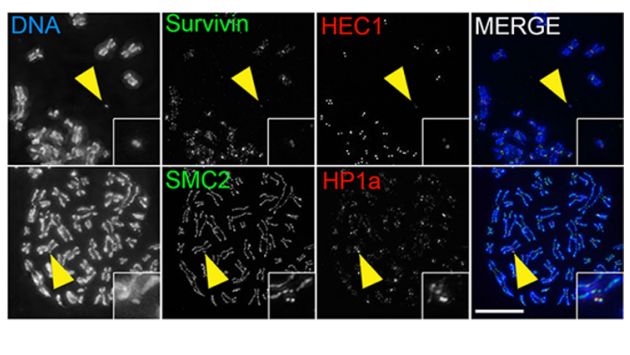

Figure 5. Hybrid HAC formation in HT1080 cells. (a) Representative FISH images of clones containing a HAC (left) and an array integration in an endogenous chromosome (right). (b, c) Screening of blasticidin-resistant clones by FISH. Diagrams represent the frequency of metaphases with HACs (black bars) and array integrations (gray bars) $(N=25)$ in HT1080 cells without (b) and with (c) CENP-A overexpression. (d) Frequency of HACcontaining clones with (CENP-A OE) and without (CENP-A WT) transient CENP-A overexpression during HAC formation. Only clones with a minimum of $10 \%$ metaphases containing HACs were considered as positive (10 vs 33\%). (e) Representative two-color oligo-FISH images showing different hybrid HACs (clone 20.CA.07-top and 20.CA.24-bottom) containing tetO (red) and lacOgal4 (green) domains. Images were captured at optimized exposure times to clearly distinguish both signals in either clone (for signal intensity comparison between clones, see Figure S2). (f) Representative image of an HT1080 cell containing HAC clone 20.CA.24 and expressing both lacI-GFP (green) and tetR-mCherry (red) fusion proteins. Merged image (right panel) represents the overlay of GFP, mCherry, and DAPI channels. (g) Frequency of HAC-containing metaphases in the indicated clones containing HACs in the presence of blasticidin and after 30 days after blasticidin washout. The HAC loss rate is indicated in red. (h) Representative immunofluorescence images on metaphase spreads of HAC clone 20.CA.24 and stained with the indicated antibodies. Scale bars $=10 \mu \mathrm{m}$.

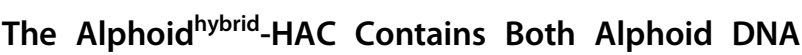
Arrays. Previous molecular analyses have revealed that HACs are formed by amplification of the input DNA that may be accompanied by structural rearrangements, generating complex structures containing alternating blocks of $\alpha$-satellite DNA and vector backbone. ${ }^{10,11,43,44}$ Because we used a hybrid $\alpha$-satellite array as input DNA for HAC formation, it was possible that the

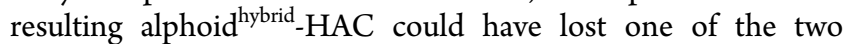
original arrays as a consequence of DNA reorganization in HT1080 cells. Therefore, we used oligo-FISH with labeled oligonucleotides that specifically recognize the tet $\mathrm{O}, \mathrm{lacO}$, and gal4 sequences to examine whether the HAC contains both $\alpha$-satellite arrays. Oligo-FISH was first developed by Matera and Ward ${ }^{45}$ to detect repetitive sequences such as specific $\alpha$-satellite DNA families. The use of small oligonucleotide probes, which show superior hybridization kinetics, has proven to be superior for faster and more sensitive FISH protocols. In our case, the repetitive nature of the hybrid HAC allows the visualization of oligonucleotide probes, as they hybridize in large numbers, thus allowing specific detection of the two different arrays in the HAC.

This experiment revealed that the HACs in both selected clones (20.CA.07 and 20.CA.24) have FISH signals for tetO and 
lacO + gal4 oligonucleotide probes (Figure 5e and Figure S1a). Therefore, the novel HACs contain both synthetic $\alpha$-satellite DNA arrays. Importantly, a control experiment using the 1C7 cell line, which contains the alphoid ${ }^{\text {tetO }}-\mathrm{HAC}^{28}$ showed a signal only for the tetO DNA array with no signal for the lacO + gal4 DNA array. This confirms the specificity of the probes used for oligo-FISH (Figure S1a).

To further confirm the presence of both synthetic arrays in the

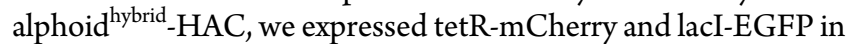
these HT1080 cell lines and then analyzed the tethering of these fusion proteins to the hybrid HACs. As expected, the results showed the presence of signals for both tetR-mCherry and lacIEGFP in interphase nuclei (Figure 5f). The signals were also detected on the HACs in metaphase chromosome spreads, where the HACs can be identified by DAPI staining (Figure S1b).

These data demonstrate the successful construction of novel synthetic HACs containing both $\alpha 21$-I-tetO and $\alpha 21$-II-lacOgal 4 synthetic alphoid-DNA arrays. Such alphoid ${ }^{\text {hybrid-HACs rep- }}$ resent a novel targeting system for future epigenetic engineering studies of the centromere.

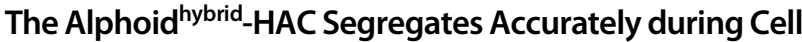
Division. Two independent clones containing alphoid ${ }^{\text {hybrid }}$-HACs (clones 20.CA.07 and 20.CA.24) were selected for further studies. In clone 20.CA.24, only $20 \%$ of the cells carry the alphoid ${ }^{\text {hybrid }}$ HAC; for this reason, we subcloned this cell line by limiting dilution to obtain a homogeneous population of HAC-containing cells. A total of 37 BS-resistant subclones were analyzed by FISH for the presence of the alphoid ${ }^{\text {hybrid-HAC. Among them, one }}$ subclone (subclone 5B10) was shown to carry single alphoid ${ }^{\text {hybrid }}$ HACs in $100 \%$ of the metaphases analyzed $(N=25)$. The other subclones showed neither HACs nor integration in chromosome arms, suggesting that only the bsr gene was integrated.

To analyze the mitotic stability of these HACs, we grew the cells in the presence and absence of BS selection for 30 days ( $\sim 30$ cell divisions) and monitored the presence of HACs by FISH. As a positive control, we included the $1 \mathrm{C} 7$ cell line, which contains the previously constructed alphoid ${ }^{\text {tetO }}-\mathrm{HAC}^{27,28}$ The daily loss rate of the HAC (R) was calculated using the formula $N_{n}=N_{0} \times(1-R) n$, where $N_{0}$ is the number of metaphase chromosome spreads showing HAC in the cells cultured under selection, $N_{n}$ is the number of HAC-containing metaphase chromosome spreads after $n$ days of culture in the absence of selection.

In 1 C 7 cells, the alphoid ${ }^{\text {tetO }}-\mathrm{HAC}$ was present in 22 out of 25 metaphases analyzed (88\%) when cells were grown in the presence of BS. In the absence of BS selection for 30 days, the alphoid $^{\text {tetO }}$-HAC was present in 20 out of 25 metaphases (80\%). Thus, the daily loss rate of the alphoid ${ }^{\text {tetO }}-\mathrm{HAC}$ was established at 0.0032 (Figure $5 \mathrm{~g}$ ). This is consistent with the values previously observed $(0.0021-0.0054) .^{27}$

The alphoid ${ }^{\text {hybrid }}$-HAC in clone 20.CA.07 showed a decrease of HAC-containing metaphases from 76 to $72 \%$ when BS selection was removed for 30 days, and the frequency of metaphases with the HAC in clone 20.CA.24-5B10 decreased from 96 to $80 \%$ after BS washout (Figure $5 \mathrm{~g}$ ). Thus, the daily rates of loss observed in clones 20.CA.07 and 20.CA.24-5B10 were 0.0018 and 0.0054 , respectively, showing a mitotic behavior similar to that of the alphoid ${ }^{\text {tetO }}$-HAC. These results indicate that the alphoid ${ }^{\text {hybrid }}$-HACs have a high mitotic stability in the absence of selection, indicating that these HACs replicate and segregate accurately during cell division.

The high mitotic stability observed for the alphoid ${ }^{\text {hybrid }}$-HACs suggests that these synthetic chromosomes contain the basic elements to maintain chromosome function. To confirm this, we performed immunofluorescence (IF) on unfixed metaphase spreads with antibodies that recognize different complexes that are important for chromosome segregation (Figure $5 \mathrm{~h}$ ). IF showed the presence of the outer kinetochore protein HEC1 on the alphoid $^{\text {hybrid }}$-HAC (Figure $5 \mathrm{~h}$ ). Other protein complexes important for centromere function were also detected on the HAC at levels similar to endogenous chromosomes: specifically, the chromosome passenger complex shown by the presence of survivin staining of the HAC, the chromosome scaffold compartment shown by SMC2 staining of the HAC, and pericentromeric heterochromatin shown by the presence of HP $1 \alpha$ in the HAC (Figure 5h). We thus conclude that the alphoid ${ }^{\text {hybrid-HAC }}$ contains a functional kinetochore resembling that of endogenous chromosomes.

The alphoid ${ }^{\text {hybrid }}$-HAC has a high mitotic stability comparable to the synthetic alphoid ${ }^{\text {tetO }}-\mathrm{HAC}$ described previously. ${ }^{27}$ Furthermore, the alphoid ${ }^{\text {hybrid }}$-HAC resembles natural chromosomes, containing the basic protein complexes necessary for chromosome structure, function, and stability.

Structural Characterization of the Alphoid hybrid_HAC Shows Contiguous Tandem Copies of the Input DNA. Because the alphoid ${ }^{\text {hybrid }}$ array forms mitotically stable HACs that resemble natural chromosomes, we went on to perform cytogenetic and molecular biology experiments to characterize the molecular organization of both alphoid ${ }^{\text {hybrid }}$-HAC clones. OligoFISH experiments on metaphase spreads clearly showed that the $\mathrm{HAC}$ in clone 20.CA.24-5B10 is larger than the HAC in clone 20.CA.07, as shown by DAPI and FISH signal intensities for both the tetO and lacOgal4 domains (Figure S2). Importantly, these results were confirmed by expressing tetR-EYFP and lacI-mCherry fusion proteins in HT1080 cells containing the alphoid ${ }^{\text {hybrid }}$ HACs. Whereas the alphoid ${ }^{\text {hybrid }}$-HAC in clone 20.CA.24-5B10 showed clear signals for both fusion proteins tethered to tetO and lacO sites, those signals were hardly visible in clone 20.CA.07 (data not shown). For this reason, we used the 20.CA.24-5B10

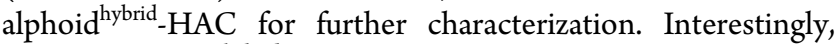
although alphoid ${ }^{\text {hybrid }}$-HAC 20.CA.07 is significantly smaller, its mitotic stability is higher compared to those of other synthetic HACs. Therefore, HAC size does not correlate in a simple manner with mitotic stability.

As shown above, FISH analysis of interphase and metaphase cells revealed the presence of lacOgal4 and tetO arrays in the alphoid $^{\text {hybrid }}$-HAC in clone 20.CA.24 (Figure 5e). We performed high-resolution fiber-FISH analysis to see how the arrays are organized in this HAC. DNA fibers were prepared from HT1080 cells containing the HAC and hybridized with either tetOspecific or a mixture of the lacO plus gal4-specific oligonucleotide probes $^{46}$ (see Materials and Methods). Representative images are shown in Figure 6a. This fiber-FISH analysis revealed alternating blocks of $\alpha 21$-I-tetO and $\alpha 21$-II-lacOgal 4 arrays. The results are consistent with those obtained by interphase FISH analysis of the 20.CA.07 alphoid ${ }^{\text {hybrid }}$-HAC (Figure S3a-c), which show $\alpha 21$-I-tetO and $\alpha 21-\mathrm{II}-\mathrm{lacOgal} 4$ blocks that are repeated in tandem.

Structural analysis of the alphoid ${ }^{\text {hybrid }}$-HACs in both clones was also carried out by Southern blot hybridization. Genomic DNA containing the alphoid ${ }^{\text {hybrid }}$-HACs was digested by SpeI endonuclease. This endonuclease cuts the vector RCA-Sat43 sequence once but does not have recognition sites in the alphoid $\alpha 21$-I-tetO or $\alpha 21$-II-lacOgal4 DNA arrays. SpeI-digested genomic DNA was separated by CHEF gel electrophoresis and hybridized with two different probes. One probe was specific to the tetO-alphoid sequence. The other was specific for both the 


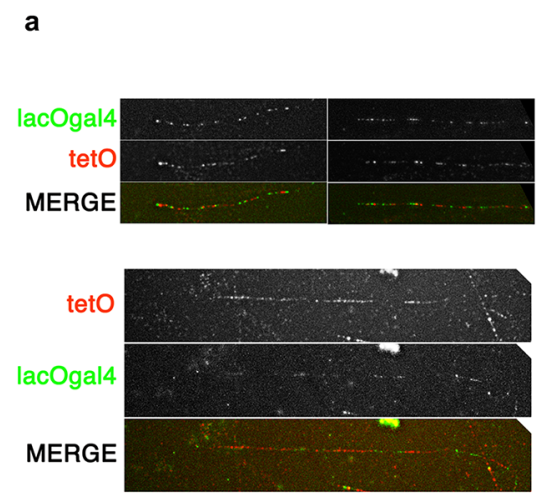

a

c

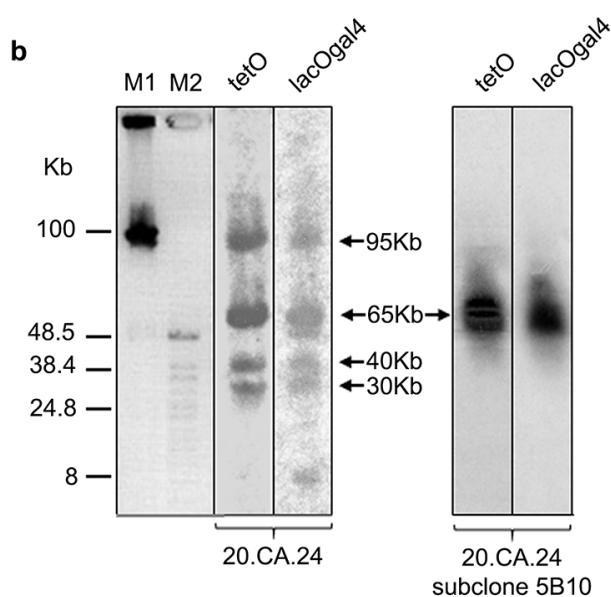

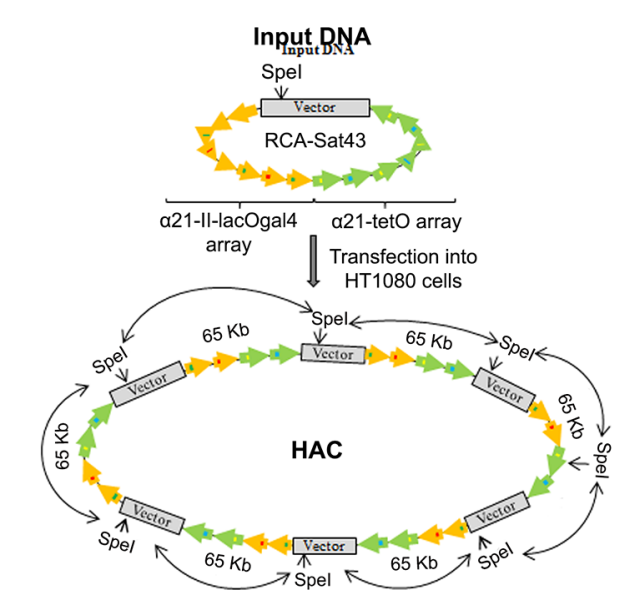

Figure 6. Structural analysis of the hybrid HAC propagated in human HT1080 cells. (a) Representative fiber-FISH images of clone 20.CA.24 HAC using oligonucleotide probes for tetO (red) and lacO + gal4 sequences (green). Different degrees of fiber stretching are shown (compare upper and lower panels). (b) Genomic DNA possessing the original HAC clone 20.CA.24 (left panel) and its subclone (5B10; right panel) were digested with SpeI endonuclease and separated by CHEF gel electrophoresis (range 10-100 kb). The SpeI recognition site is present once in the RCA-SAT43 vector at position 812 but not in the hybrid array. The transferred membrane was hybridized with radioactively labeled tetO-specific or lacO + gal4-specific probes. The 5B10 subclone has a HAC with a remarkably conserved array. Arrows indicate fragments of 95, 65, 40, and $30 \mathrm{~kb}$ in size that are specific to both probes. (c) Diagram illustrating multimerization of input DNA during de novo HAC formation in human HT1080 cells. Input DNA consists of $65 \mathrm{~kb}$ hybrid array and 10,209 bp RCA-Sat43 vector sequence.

lacO and gal4 sequences (see Materials and Methods for details). If the HACs were formed by simple concatenation involving rolling-circle amplification of the input DNA and had not undergone structural rearrangements, only one band of $65 \mathrm{~kb}$ in size ( $\sim 55 \mathrm{~kb}$ alphoid array plus $\sim 10 \mathrm{~kb}$ vector part) would be observed on the Southern blot after SpeI digestion. For the original clone 20.CA.24 (containing 25\% of HAC-containing metaphases; see Figure 5), four major bands of 95, 65, 40, and $30 \mathrm{~kb}$ were detected with the tetO probe, and a similar profile with two additional minor bands of 60 and $8 \mathrm{~kb}$ was observed with the lacOgal4 probe (Figure 6b). For clone 20.CA.07, two major bands of 95 and $65 \mathrm{~kb}$ were detected with the tetO and lacOgal4 probes (Figure S3d).

Unexpectedly, for 20.CA.24 subclone 5B10, only one major band of $65 \mathrm{~kb}$ in size was observed $(\sim 55 \mathrm{~kb}$ array plus $\sim 10 \mathrm{~kb}$ vector). These results indicate that the original clones 20.CA.24 and 20.CA.07 are likely a mixture of the cells with different-sized HACs. In contrast, the 5B10 subclone contains only one HAC with a regular structure containing alternating tet $\mathrm{O}$ and lacOgal4. This could suggest a rolling circle amplification mechanism of formation of this HAC. These results are in agreement with the alternating organization of $\alpha 21$-I-tetO and $\alpha 21$-II-lacOgal4 DNA blocks observed by fiber-FISH analysis (Figures 6a). Taking these data together, a proposed diagram of the hybrid HAC in subclone 5B10 is presented in Figure 6c.

Interestingly, this novel alphoid ${ }^{\text {hybrid }}$-HAC (subclone 5B10) had much more regular structure than the original synthetic alphoid $^{\text {tetO }}$-HAC (subclone AB2.218.21). ${ }^{27}$ Southern blot hybridization of the SpeI-digested genomic DNA harboring the alphoid $^{\text {tetO }}$-HAC revealed 15 fragments of different size, indicating complex rearrangements of $50 \mathrm{~kb}$ input alphoid ${ }^{\text {tetO }}$ DNA array during HAC formation. ${ }^{43}$ It also contained a large region from the long arm of chromosome 13, suggesting that at one point during its formation it may have integrated into that chromosome. It is possible that the more regular structure of the alphoid ${ }^{\text {hybrid }}$-HAC may be due to a more efficient process of de novo HAC formation, possibly as a result of CENP-A overexpression.

These results show that de novo alphoid ${ }^{\text {hybrid }}$-HAC formation was accompanied, as expected, by amplification of the input DNA molecule but that amplification can occur without gross structural rearrangements. Future experiments will determine whether this is due to $\mathrm{HAC}$ formation in the presence of overexpressed CENP-A. 
a

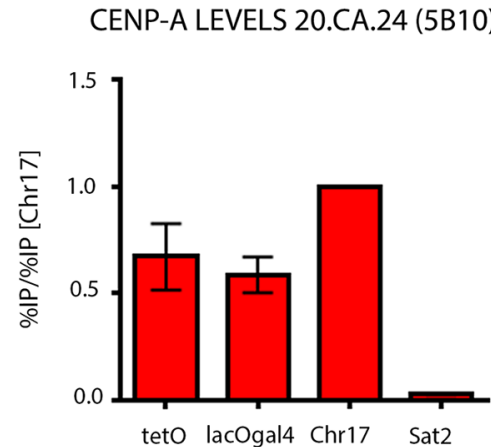

$$
\text { b }
$$

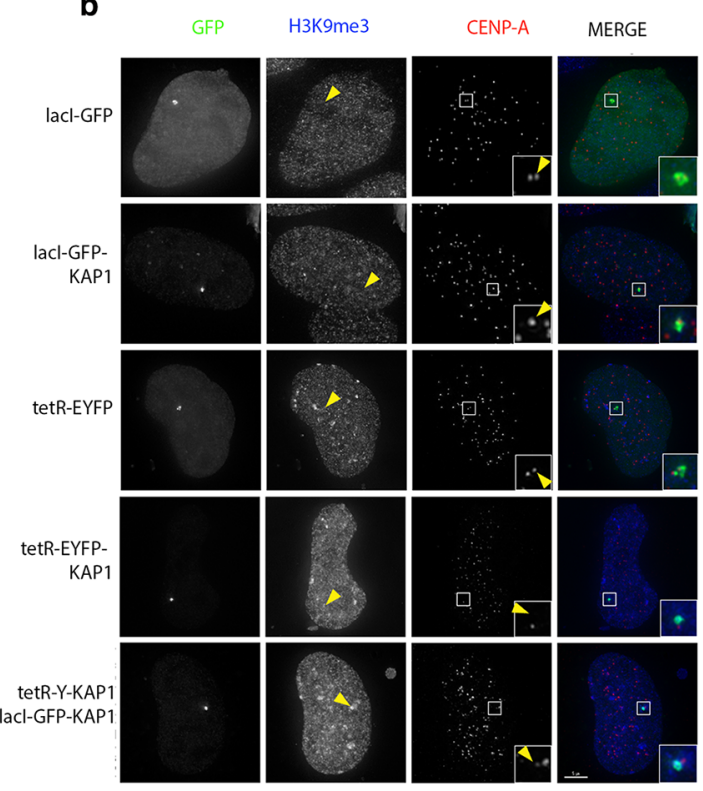

c

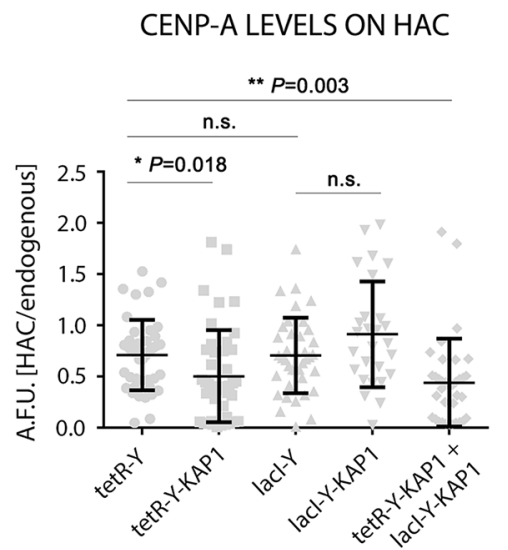

CENP-A LEVELS ON HAC 
a

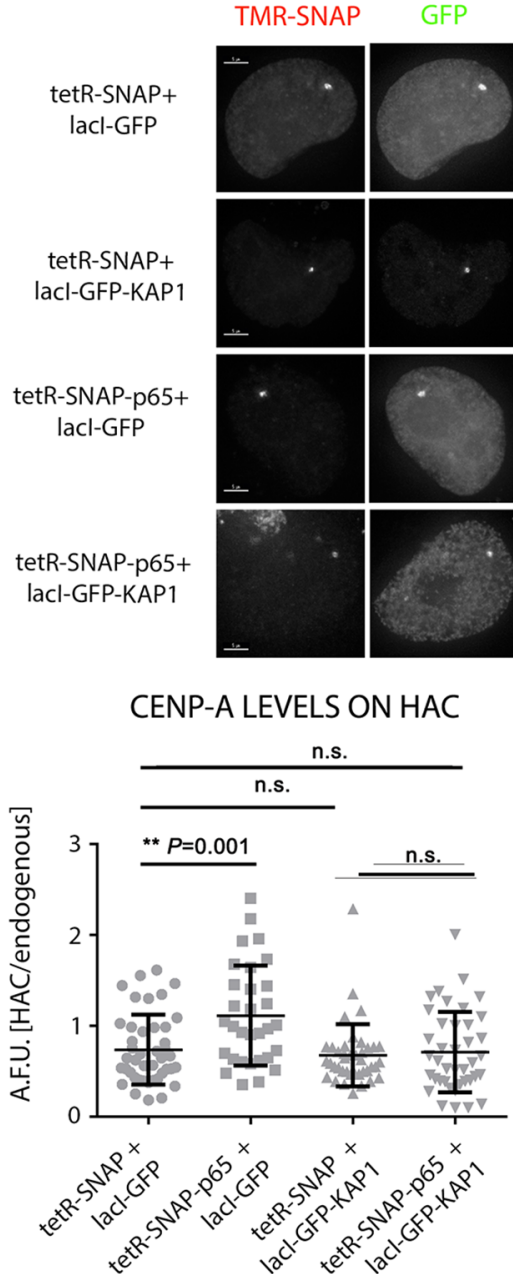

H3K9me3

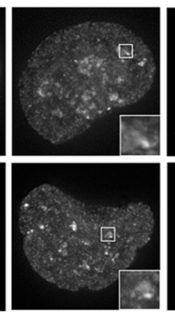

CENP-A

MERGE 1

MERGE 2

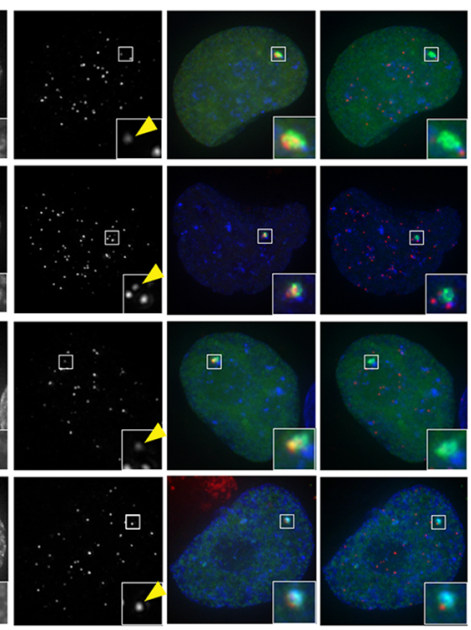

C

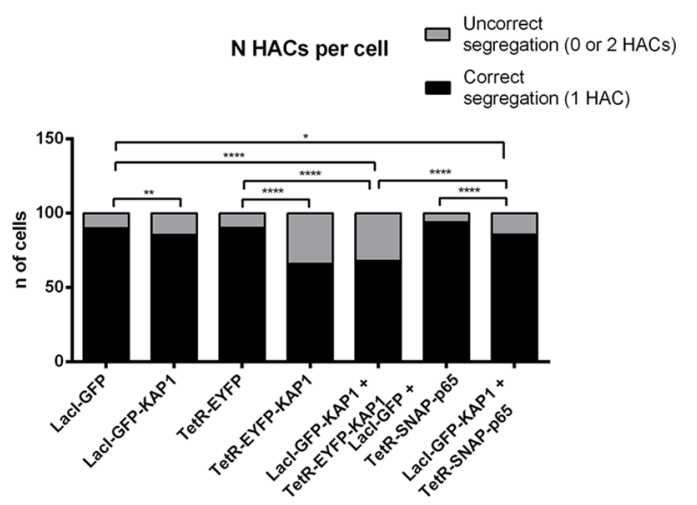

d
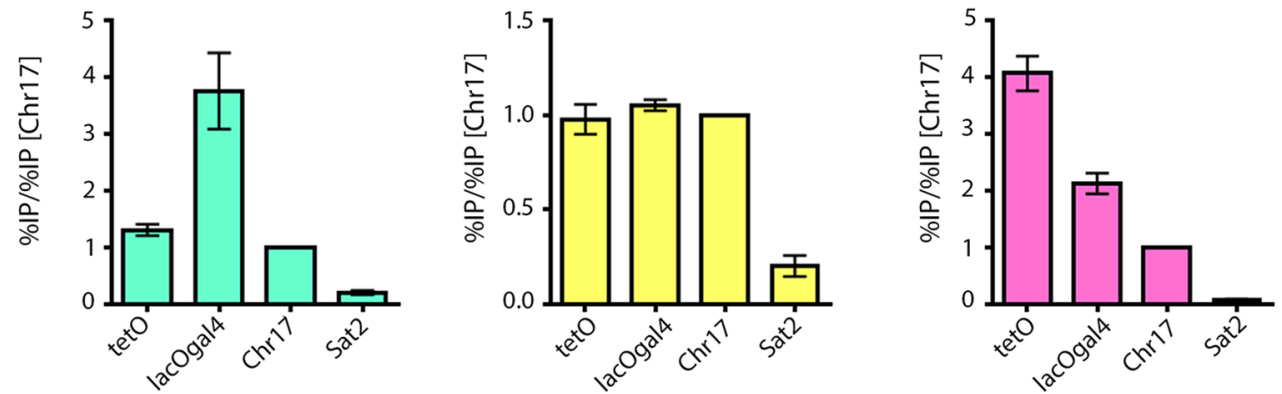

Figure 8. Alphoid ${ }^{\text {hybrid }}$ HAC shows epigenetically distinct centromeric domains. (a) Representative images of HT1080-5B10 cells expressing the indicated tetR (first panel) and lacI-fusion proteins (second panel) and stained with antibodies recognizing H3K9me3 (third panel) and CENP-A (fourth panel). Merged images represent the overlay of TMR-SNAP, GFP, and H3K9me3 (MERGE 1; fifth panel) and GFP, H3K9me3 and CENP-A (MERGE 2; sixth panel). (b) Quantification of HAC-associated CENP-A staining in individual cells transfected with the indicated fusion proteins and plotted as A.F.U. Solid bars indicate the medians, and error bars represent the s.e.m. $n=$ two independent experiments for each time point and staining. Asterisks indicate a significant difference $\left(* * P<0.01\right.$; Mann-Whitney test). (c) Quantification of alphoid ${ }^{\text {hybrid }}$ HAC copy-numbers as determined by counting the GFP and/or TMR-SNAP spot in interphase nuclei of cells transfected with the indicated fusion proteins. Data represent the mean (and s.e.m.) of three independent assays of each time point after doxycycline washout $\left(n=1,000\right.$ nuclei per condition; $* P<0.05, * * P<0.0001 ; \chi^{2}$-test). (d) ChIP-qPCR analysis in HT1080-5B10 cells using the indicated antibodies. The $\alpha 21$-I-tetO (tetO), $\alpha 21$-II-lacOgal4 (lacOgal4) hybrid HAC domains, the satellite D17Z1 (Chr17), and the degenerate satellite type-II (Sat2) repeats were assessed. Scale bars $=10 \mu \mathrm{m}$.

NF-KB p65 to the $\alpha 21$-I-tetO domains and KAP1 to the $\alpha 21$-IIlacOgal4 domains as tetR-SNAP and lacI-GFP fusion proteins, respectively. Tethering of tetR-SNAP-p65 together with lacI-GFP significantly increased CENP-A levels on the alphoid ${ }^{\text {hybrid }}$-HAC compared to that of a control tethering of tetR-SNAP with lacIGFP (Figures $8 \mathrm{a}$ and $\mathrm{b}$ ). As previously reported for the alphoid ${ }^{\text {tetO }}$ HAC, ${ }^{29}$ tethering tetR-SNAP-p65 to the alphoid ${ }^{\text {hybrid }}$-HAC increases levels of $\mathrm{H} 3 \mathrm{~K} 9 \mathrm{ac}$ (Figure S4b). In parallel experiments, 
tethering lacI-GFP-KAP1 to the alphoid ${ }^{\text {hybrid }}$-HAC increased the H3K9me3 level (Figure S4a). Simultaneous tethering of lacIGFP-KAP1 together with tetR-SNAP maintained the CENP-A level similar to that observed in controls (Figure $8 \mathrm{a}$ and $\mathrm{b}$ ).

Strikingly, simultaneous tethering of tetR-SNAP-p65 with lacIGFP-KAP1 seems to balance the levels of H3K9ac and $\mathrm{H} 3 \mathrm{~K} 9 \mathrm{me} 3$ (Figure S4), and the levels of CENP-A are maintained similar to those observed in controls (Figure 8a and b). Thus, although there does appear to be cross-talk between the two domains of the HAC, kinetochore function is preserved as demonstrated by the proper segregation of the alphoid ${ }^{\text {hybrid }}$-HAC when these fusion proteins were tethered together (Figure 8c).

The epigenetic environment in the centromere is important for kinetochore assembly and maintenance. ${ }^{23}$ To study the epigenetic differences between the different centromeric domains in the alphoid ${ }^{\text {hybrid }}$-HAC, we performed ChIP-qPCR experiments pulling down different histone $\mathrm{H} 3$ modifications typically associated with centromeric chromatin, such as H3K4me2, H3K36me2, and H3K9me3 (Figure 8d). The results showed that there is no difference in the amount of the heterochromatin marker $\mathrm{H} 3 \mathrm{~K} 9 \mathrm{me} 3$ between $\alpha 21$-I-tetO and $\alpha 21$-II-lacOgal4 domains or between the alphoid ${ }^{\text {hybrid }}$-HAC and the centromere of the endogenous chromosome 17 (Figure 8d). Thus, the level of heterochromatin is similar between the alphoid ${ }^{\text {hybrid }}$-HAC centromere and endogenous centromeres. The lower level of $\mathrm{H} 3 \mathrm{~K} 9 \mathrm{me} 3$ in the Sat 2 region could be explained by the lower level of heterochromatin in HT1080 cells (Figure 8d). ${ }^{36,47}$

We also observed different levels of chromatin marks associated with actively transcribed chromatin on the $\alpha 21-\mathrm{I}$ tetO and $\alpha 21$-II-lacOgal4 arrays. Whereas $\mathrm{H} 3 \mathrm{~K} 4 \mathrm{me} 2$ is preferentially enriched on the $\alpha 21$-II-lacOgal 4 arrays, $\mathrm{H} 3 \mathrm{~K} 36 \mathrm{me} 2$ was preferentially enriched on the $\alpha 21$-I-tetO arrays (Figure $8 \mathrm{~d}$ ). The different localizations of $\mathrm{H} 3 \mathrm{~K} 4 \mathrm{me} 2$ and $\mathrm{H} 3 \mathrm{~K} 36 \mathrm{me} 2$ on centrochromatin suggest that these histone modifications may play different roles in kinetochore assembly and/or maintenance. Previous results showed that removing $\mathrm{H} 3 \mathrm{~K} 4 \mathrm{me} 2$ from the alphoid ${ }^{\text {tetO }}$-HAC centromere disrupts kinetochore maintenance and function. ${ }^{33}$ The present results suggest that it will be important in the future to explore the role of $\mathrm{H} 3 \mathrm{~K} 36 \mathrm{me} 2$ in centrochromatin in greater detail.

Our proof of principle experiments reveal that the

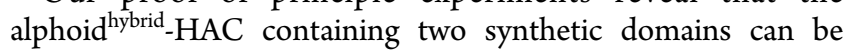
manipulated for epigenetic engineering studies. Our results with this novel HAC reinforce the model that centromeres are assembled and maintained by a specific chromatin environment. Moreover, our experiments combining the opposing chromatin modifiers p65 and KAP1 revealed that, although there is crosstalk between the kinetochore and heterochromatin domains, induced heterochromatin disrupts the centromere only when it is nucleated within centrochromatin. Inducing heterochromatin in the pericentromeric domains did not disrupt the kinetochore, possibly due to the presence of specific histone modifications and/or active RNAP II transcription at centrochromatin that protects it from heterochromatin spreading.

\section{CONCLUSIONS}

We developed and characterized a novel synthetic alphoid ${ }^{\text {hybrid }}$ HAC (human artificial chromosome) containing two distinct alphoid DNA arrays. The two synthetic arrays can remain remarkably preserved during HAC formation in contrast to the gross rearrangements observed with the previous generation of alphoid $^{\text {tetO }}$ HACs. The alphoid ${ }^{\text {hybrid }}$-HAC allows simultaneous independent targeting of chromatin modifiers to different centromeric compartments using independent tetO and lacO/ gal4 targeting systems, and our preliminary experiments reveal that there is some cross-talk between the chromatin in two arrays. The alphoid ${ }^{\text {hybrid }}$-HAC may be used in future studies to clarify whether the endogenous CENP-A chromatin is continuous or interrupted with blocks of heterochromatin in natural human chromosomes.

\section{MATERIALS AND METHODS}

Construction of $\alpha 21-1-$ tetO and $\alpha 21-\mathrm{II}-$ lacOgal4 Alphoid Arrays by Rolling-Circle Amplification (RCA) Followed by Transformation-Associated Recombination (TAR) in Yeast. A method to rapidly convert any desirable DNA fragment, as small as $100 \mathrm{bp}$, into long tandem DNA arrays up to $140 \mathrm{~kb}$ in size is described in detail in a previous publication. ${ }^{48}$ The method includes rolling-circle phi29 amplification (RCA) of the sequence in vitro and assembly of the RCA products in vivo by homologous recombination in the yeast Saccharomyces cerevisiae. In our case, for the RCA reaction we used a $343 \mathrm{bp}$ 21-I alphoid dimer from high-order repeats (HORs) of chromosome 21 containing CENP-B boxes and a 2,078 bp synthesized 21-II-lacOgal4/CENPB minus-12-mer. In one of the monomers of the 21-I alphoid dimer, a CENP-B box was replaced by a $42 \mathrm{bp}$ tetO motif (see Figure 1 ). In the 21-II alphoid 12-mer, four gal4 sequences (each $21 \mathrm{bp}$ in size) and four lacO sequences (each $36 \mathrm{bp}$ in size) were incorporated (see Figure 2). RCA reactions were performed using an Amersham TempliPhi kit according to the manufacturer's instructions except that reactions were scaled up to $100 \mu \mathrm{L}$ and were spiked with a template-specific primer mix to a final concentration of $2 \mathrm{pmol} / \mu \mathrm{L}$ (Figure 3a). Reaction products were phenol/ chloroform extracted and ethanol precipitated prior to recombinational cloning. As a second step, the length of the resulting RCA products was extended by transformation-associated homologous recombination (TAR) in yeast ${ }^{25,26,49}$ using a targeting RCASat43 vector ${ }^{24}$ (Figure 3B). The RCA-Sat43 vector (10,209 bp in size) contains YAC (HIS3, CEN6, ARSH4) and BAC ( $\mathrm{Cm}$, ori F $)$ cassettes as well as a mammalian selectable marker (BSR). Also, the RCA-Sat43 vector contains the appropriate alphoid satellite hooks of $\sim 40 \mathrm{bp}$ each. Before yeast transformation, the vector was linearized by MluI to release targeting hooks. The highly transformable Saccharomyces cerevisiae strain VL6-48N (MATalpha, his3- $\Delta 200$, trp1- $\Delta 1$, ura3- $\Delta 1$, lys2, ade2-101, met14), which has HIS3 and URA3 deletions, was used for transformation. Conditions for spheroplast transformation were described previously. ${ }^{50}$ Typically, each transformation used $2-3 \mu \mathrm{g}$ of RCA product and $0.02 \mu \mathrm{g}$ of the linearized vector. Under such conditions, 200-1000 His+ transformants were usually obtained. As a third step, yeast transformants were combined into pools, and then purified genomic DNA from the yeast clones was electroporated into Escherichia coli cells (DH10B, Invitrogen). The insert size of $\alpha 21$-II-lacOgal 4 arrays was determined by CHEF (Bio-Rad) after AvaII digestion, which releases an $\sim 6.5 \mathrm{~kb}$ vector fragment and an array plus $\sim 3.5 \mathrm{~kb}$ vector fragment. Representative CHEF analysis of 13 BACs is shown (Figure 4a). Digestion of the $\alpha 21$-II-lacOgal 4 arrays by AlwNI reveals 12-mers of $2,078 \mathrm{bp}$ in size (Figure $4 \mathrm{~b}$ ). The insert size of $\alpha 21$-I-tetO arrays was determined by CHEF (Bio-Rad) after NotI digestion that releases an $\sim 2 \mathrm{~kb}$ fragment of the RCA-Sat 43 vector and an array plus $\sim 7 \mathrm{~kb}$ vector fragment. Representative $\mathrm{CHEF}$ analysis of one BAC is shown (Figure 4c). Digestion of the $\alpha 21$-I-tetO arrays by EcoRI reveals 2-mers of 343 bp in size (Figure 4d). 
Construction of the Hybrid tetO-CENPB+/lacOgal4CENPB- Array by Recombinational Cloning in Yeast. A general scheme of construction of the synthetic hybrid tetOCENPB+/lacOgal4-CENPB- array is presented in Figure 3c. The $\sim 40 \mathrm{~kb} \alpha 21$-II-lacOgal4 array (Figure $4 \mathrm{a}$; lane 12) and $\sim 40 \mathrm{~kb}$ $\alpha 21$-I-tetO array (Figure 4c) were chosen for construction of the hybrid tetO-CENPB+/lacOgal4-CENPB- array. Restriction of $\mathrm{BAC}$ DNAs was done by endonucleases that cleave the molecule at insert/vector junctions and by an endonuclease that cuts the vector part completely. The $40 \mathrm{~kb} \alpha 21$-II-lacOgal 4 array was released by digestion with $\mathrm{MspI} / \mathrm{NsiI}$ endonucleases (33 MspI recognition sites are present in the RCA-Sat 43 vector). The $40 \mathrm{~kb}$ $\alpha 21-\mathrm{I}-$ tetO array was released by digestion with $\mathrm{MseI} / \mathrm{SpeI} / \mathrm{NheI}$ endonucleases (57 MseI recognition sites are present in the RCA-Sat43 vector). The $\alpha 21$-II-lacOgal4 and $\alpha 21$-I-tetO arrays were gel-purified after CHEF separation, mixed with MluIdigested RCA-Sat43 vector (the MluI site is present between the hooks and not present in the arrays) and transformed into Saccharomyces cerevisiae strain VL6-48N. Typically, $0.5 \mu \mathrm{g}$ of each array and $0.02 \mu \mathrm{g}$ of the linearized RCA-Sat 43 vector were used for one transformation. Under such conditions, 100-300 His+ transformants were usually obtained. Next, genomic DNA from yeast clones washed from plates was isolated and electroporated into Escherichia coli cells (DH10B, Invitrogen). Because homology between $\alpha 21$-II-lacOgal 4 and $\alpha 21$-I-tetO arrays is $\sim 70-74 \%$, the percentage of the hybrid arrays was very low, i.e., $\sim 0.1 \%$. The insert size of the entire hybrid arrays in the BACs was checked by MspI digestion. The size of the $\alpha 21$-I-tetO part in the hybrid arrays was checked by MseI digestion (this endonuclease completely digests a vector part and a $\alpha 21$-IIlacOgal 4 array). The size of the $\alpha 21$-II-lacOgal 4 array in the hybrid arrays was checked by MsII or AlwI (these endonucleases digest a vector part and the $\alpha 21$-I-tetO array). A BAC containing a hybrid array consisting of $\sim 25 \mathrm{~kb} \alpha 21$-II-lacOgal4 array and $\sim 30 \mathrm{~kb} \alpha 21$-I-tetO array was chosen for further HAC development experiments (Figure 4e).

Southern Blot Hybridization Analysis. Southern blot hybridization was performed with a ${ }^{32} \mathrm{P}$-labeled probe as described previously ${ }^{51}$ with minor changes. Genomic DNA was prepared in agarose plugs and restriction-digested by SpeI in the buffer recommended by the manufacturer. The digested DNA was CHEF (CHEF Mapper, Bio-Rad) separated (autoprogram, 5-150 kb range, $16 \mathrm{~h}$ transfer), transferred to membrane (Amersham Hybond-N+), and blot-hybridized with an 82 bp probe for tetO and a $74 \mathrm{bp}$ probe for lacO and gal4. DNA sequences for the probes were amplified by PCR using the primers and synthetic DNA fragment as a template (tetO_south_21_M: 5'-TTTGTGGAAGTGGACATTTACTAGCAGCAĞAGCTCTCCCTATCAGTGATAGAGACTAGCCCATAAAAATAGACAGAAGCATT-3', tetO_south_21_F1: 5'-TTTGTGGAAGTGGACATTTC-3', tetO_south_21_-R1: 5'-AATGCTTCTGTCTATTTTTA-3'; lacO_south3_M: 5'-TGTGGAAGTGGACATTTCGACCACATGTGGAATTGTGAGCGGATAACAATTTGTGGCCCATAAAAATAGACAGA-3', lacO_south3_F1: 5'-TGTGGAAGTGGACATTTCGA-3', lacO_south3_R1: 5'-TCTGTCTATTTTTATGGGCC-3'; gal $\overline{4}$ south 1 _.M: 5'-AATGGACATTTCGACGGAGGACAḠTCCTCCGTCGACGGAGGACAGTCCTCCGCATAAAATCTA-3', Gal4_south1_F1: 5'-AATGGACATTTCGACG-3', gal4_south1_- 1 1: 5'-TAGATTTTATGCGGAG-3'). The blot was incubated for $2 \mathrm{~h}$ at $65{ }^{\circ} \mathrm{C}$ for prehybridization in Church's buffer ( $0.5 \mathrm{M}$ Na-phosphate buffer containing $7 \%$ SDS and $100 \mu \mathrm{g} / \mathrm{mL}$ of unlabeled salmon sperm carrier DNA). The labeled probe was heat denatured in a boiling water bath for $5 \mathrm{~min}$ and snap-cooled on ice. The probe was added to the hybridization Church's buffer and allowed to hybridize for $48 \mathrm{~h}$ at $65^{\circ} \mathrm{C}$. Blots were washed twice in $2 \times$ SSC $(300 \mathrm{mM}$ $\mathrm{NaCl}, 30 \mathrm{mM}$ sodium citrate, $\mathrm{pH} 7.0$ ), $0.05 \%$ SDS for $10 \mathrm{~min}$ at room temperature, then twice in $2 \times$ SSC, $0.05 \%$ SDS for $5 \mathrm{~min}$ at $60{ }^{\circ} \mathrm{C}$. Blots were exposed to X-ray film for 5 days at $-80{ }^{\circ} \mathrm{C}$.

Cell Culture, Transfections, and HAC Formation. Human HT1080 cells were maintained in DMEM supplemented with $10 \%$ FBS (Invitrogen) plus $100 \mathrm{U} / \mathrm{ml}$ penicillin $\mathrm{G}$ and $100 \mu \mathrm{g} / \mathrm{mL}$ of streptomycin sulfate (Invitrogen). Cells were grown at $37^{\circ} \mathrm{C}$ in $5 \% \mathrm{CO}_{2}$ in a humidified atmosphere. Transfections were performed using Xtremegene- 9 (Roche) following the manufacturer's instructions. In brief, for transfections of cells growing in $10 \mathrm{~cm}$ dishes, transfection complexes containing $15 \mu \mathrm{L}$ of Xtremegene-9 reagent and $5 \mu \mathrm{g}$ of plasmid DNA were prepared in $500 \mu \mathrm{L}$ of OptiMEM (Invitrogen). After $20 \mathrm{~min}$ of incubation at room temperature, $500 \mu \mathrm{L}$ of transfection complexes was added dropwise in $10 \mathrm{~mL}$ of media. After $24 \mathrm{~h}$, transfected cells were selected adding $4 \mu \mathrm{g} / \mathrm{mL}$ of blasticidin $\mathrm{S}$ (Sigma) and grown for 2-3 weeks until separate resistant colonies were present. Resistant colonies were isolated by trypsinization in cloning cylinders (Thermo Scientific). Isolated clones were expanded in the presence of $4 \mu \mathrm{g} / \mathrm{mL}$ of blasticidin $\mathrm{S}$. For cotransfection experiments with the CENP-A-SNAP plasmid, $5 \mu \mathrm{g}$ of plasmid DNA was added to the transfection reaction.

Fluorescent in Situ Hybridization (FISH). Samples were processed as previously described by us. ${ }^{46}$ In brief, metaphase chromosomes were obtained following standard procedures: $3 \mathrm{~h}$ before harvesting, cells were treated with Colcemid (Invitrogen) at a final concentration of $0.2 \mu \mathrm{g} / \mathrm{mL}$. They were then resuspended in warm hypotonic solution $(0.075 \mathrm{M} \mathrm{KCl})$ for $10 \mathrm{~min}$ at $37{ }^{\circ} \mathrm{C}$ and fixed in methanol:acetic acid (3:1). To obtain stretched chromatin fibers, $2 \mathrm{~mL}$ of a cell culture was centrifuged, and the pellets were washed in $1 \times$ PBS. Pellets were resuspended in $1 \times$ PBS to reach a final concentration of $2 \times 10^{6}$ cells $/ \mathrm{mL}$ and spread on slides. Once the slides were mounted on the Shandon Sequenza cover plates (Thermo Scientific), DNA fibers were released applying a lysis solution $(0.07 \mathrm{M} \mathrm{NaOH}$ in ethanol $)$ and fixed in methanol. Slides were kept at $-20{ }^{\circ} \mathrm{C}$ until they were processed for FISH.

The RCA-Sat43 vector backbone was used as DNA probe. BAC DNA extraction was performed using the EndoFree Plasmid Maxi kit (Qiagen GmBh; Hilden, Germany) following the manufacturer's instructions. BAC DNA was labeled by Nick translation using Spectrum Green dUTPs (Abbott Molecular) following the manufacturer's instructions. Probes were mixed with $10 \mu \mathrm{g}$ of Cot 1 DNA (Invitrogen; Carlsbad, USA), ethanol precipitated, and resuspended in $1 \times$ hybridization buffer $(50 \%$ formamide, $1 \times$ SSC, and $10 \%$ dextran sulfate) to a final concentration of $40 \mathrm{ng} / \mu \mathrm{L}$. For oligo-FISH experiments, oligonucleotides recognizing the tetO sequence ( $5^{\prime}$ - ACTAGCAGCAGAGCTCTCCCTATCAGTGATAGAGACTAG-3') labeled with Digoxigenin, and oligonucleotides recognizing both lacO (5'- CATGTGGAATTGTGAGCGGATAACAATTTGTGG-3') and Gal4 (5'- TCGACGGAGGACAGTCCTCCG-3') sequences labeled with Biotin were synthesized (Sigma). Oligonucleotides were mixed at $100 \mathrm{ng} / \mu \mathrm{L}$ and resuspended in $2 \times$ hybridization buffer (Cellay Inc.), $10 \times$ blocking reagent (Cellay Inc.), and $50 \mu \mathrm{g} / \mathrm{mL}$ of salmon sperm DNA (Sigma).

FISH was carried out following standard procedures. ${ }^{46}$ Briefly, the DNA probe was denatured at $80{ }^{\circ} \mathrm{C}$ for $5 \mathrm{~min}$ and preannealed at $37{ }^{\circ} \mathrm{C}$ for $30 \mathrm{~min}$. Slides were denatured in 
$70 \%$ formamide $/ 2 \times \mathrm{SSC}$ at $70{ }^{\circ} \mathrm{C}$ for $1 \mathrm{~min}$ and hybridized in a humid chamber at $37{ }^{\circ} \mathrm{C}$ overnight. Slides were washed in $0.7 \times \mathrm{SSC} / 0.03 \% \mathrm{NP} 40$ for $1 \mathrm{~min}$ at $70{ }^{\circ} \mathrm{C}$ and in $2 \times \mathrm{SSC} / 0.03 \%$ $\mathrm{NP} 40$ for $2 \mathrm{~min}$ at room temperature. For Oligo-FISH experiments, slides were denatured in $70 \%$ formamide $/ 2 \times S S C$ at $70^{\circ} \mathrm{C}$ for $1 \mathrm{~min}$ and hybridized in a humid chamber at $37^{\circ} \mathrm{C}$ for $2 \mathrm{~h}$. Slides were thereafter washed in $20 \%$ formamide/ $2 \times$ SSC for $5 \mathrm{~min}$ and in $2 \times \mathrm{SSC} / 0.1 \%$ Tween- 20 for $5 \mathrm{~min}$ at $37{ }^{\circ} \mathrm{C}$. Oligonucleotide probes were detected with rhodamine-conjugated antidigoxigenin (Roche) and fluorescein-conjugated streptavidin (Vector Laboratories). Slides were mounted with Vectashield (Vector Laboratories) containing 4',6-diamidino-2phenylindole (DAPI) for chromosome counterstaining.

Indirect Immunofluorescence Staining and Microscopy. Indirect immunofluorescence staining of cells fixed in $4 \%$ formaldehyde $/ 1 \times$ PBS was performed following standard procedures. Immunofluorescence on unfixed metaphase spreads was performed as described previously. ${ }^{9}$ The following antibodies were used: mouse anti-CENP-A (clone A1, 1:500), mouse anti-HP1 $\alpha$ (Millipore MAB3584, 1:1000), mouse anti-HEC1 (abcam AC3612, 1:1000), rabbit anti-SMC2 (A. Losada, 1:1000), rabbit anti-Survivin (Cell Signaling, 1:400), rabbit anti-H3K9me3 (abcam 8898; 1:200), and rabbit anti-H3K9ac (B. Turner, 1:200).

Microscope images were acquired on a DeltaVision Core system (Applied Precision) using an Olympus IX-71 inverted microscope stand with an Olympus UPlanSApo 100× oil immersion objective (numerical aperture (NA) 1.4) and an LED light source. Camera (Photometrics Cool Snap HQ), shutter, and stage were controlled through SoftWorx (Applied Precision). Z-series were collected with a spacing of $0.2 \mu \mathrm{m}$, and image stacks were subsequently deconvolved in SoftWorx. For CENP-A signal quantification, a custom-made macro in ImageJ (National Institutes of Health, Bethesda, MD) modified from Bodor et al. ${ }^{52}$ was used.

Chromatin Immunoprecipitation and Quantitative PCR (ChIP-qPCR). Exponentially growing cells were washed in D-PBS (Gibco) and subsequently harvested with TrypLE Express (Gibco). Cells were resuspended in D-PBS up to a concentration of $1 \times 10^{6}$ cells $/ \mathrm{ml}$ and cross-linked in a final $1 \%$ formaldehide solution (Sigma) for $5 \mathrm{~min}$ at room temperature, followed by quenching with $2.5 \mathrm{M}$ glycine for $5 \mathrm{~min}$ at room temperature. Cells at a concentration of $5 \times 10^{6}$ cells $/ \mathrm{ml}$ were lysed in lysis buffer (10 mM Tris $\mathrm{pH} 8.0 ; 10 \mathrm{mM} \mathrm{NaCl}$; $0.5 \% \mathrm{NP}-40)$ containing protease inhibitors $(1 \mu \mathrm{g} / \mathrm{mL}$ of CLAP; $0.5 \mu \mathrm{g} / \mathrm{mL}$ of Aprotinin; $1 \mathrm{mM}$ PMSF) for $10 \mathrm{~min}$ on ice. Nuclei were briefly washed in lysis buffer with protease inhibitors in $300 \mu \mathrm{L}$ of dilution buffer 1 (50 mM Tris $\mathrm{pH} 8.0 ; 2$ mM EDTA; $0.2 \%$ SDS; $134 \mathrm{mM} \mathrm{NaCl} ; 0.88 \%$ Triton X-100; $0.088 \%$ $\mathrm{Na}$-deoxycholate). Chromatin was sheared by sonication in a Bioruptor sonicator (Diagenode) for 14 cycles (30s ON/30S $\mathrm{OFF})$ at high setting and $4{ }^{\circ} \mathrm{C}$. Supernatant products of sonication were diluted with $300 \mu \mathrm{L}$ of dilution buffer $1,500 \mu \mathrm{L}$ of dilution buffer 2 (50 mM Tris pH 8.0; $167 \mathrm{mM} \mathrm{NaCl} ; 1.1 \%$ Triton X-100; $0.11 \%$ Na-deoxycholate), and $500 \mu \mathrm{L}$ of RIPA buffer containing $150 \mu \mathrm{L}$ of $\mathrm{NaCl}$ (RIPA-150) and protease inhibitors. Antimouse IgG Dynabeads (Invitrogen) were coated with the relevant antibodies for $6 \mathrm{~h}$ with RIPA-150/0.5\% BSA at $4{ }^{\circ} \mathrm{C}$ and washed twice with RIPA- $150 / 0.5 \%$ BSA, and $500 \mu \mathrm{L}$ of sheared chromatin was incubated with the beads at $4{ }^{\circ} \mathrm{C}$ overnight. Beads were afterward washed twice with RIPA-150 and RIPA buffer containing $500 \mathrm{mM} \mathrm{NaCl}$ (RIPA-500) and a final wash with $\mathrm{TE} \mathrm{pH}$ 8.0. Antibody/chromatin complexes were de-cross-linked with 10\% Chelex-100 resin (BioRad) in water at $93{ }^{\circ} \mathrm{C}$ and treated with RNase A and Proteinase K. DNA was subsequently recovered by aliquoting $60 \mu \mathrm{L}$ of the supernatant in a new eppendorf tube. ChIPed DNA was subjected to RT-PCR using a SYBR Green Master Mix (Roche) using the following oligonucleotides: tetO-Fw (5'-CCACTCCCTATCAGTGATAGAGAA-3'), tetO-Rv (5'-TCGACTTCTGTTTAGTTCTGTGCG-3') for the $\alpha 21$-I-tetO domain of the hybrid HAC, lacOgal4-Fw (5'-TATGGTGTCGACGGAGGACA-3'), and lacOgal4-Rv (5'-CCGCTCACAATTCCACATGTG-3') for the $\alpha 21$-II-lacOgal4 domain of the hybrid HAC, chr17-Fw ( $5^{\prime}$-TTGTGGTTTGTGGTGGAAAA-3') and chr17-Rv $\left(5^{\prime}\right.$-CTCAAAGCGCTCCAAATCTC-3') for the alphoid ${ }^{\text {chr17 }}$ array, bsr-Fw (5'-CAGGAGAAATCATTTCGGCAGTAC-3') and bsr-Rv ( $5^{\prime}$-TCCATTCGAAACTGCACTACCA- $\left.3^{\prime}\right)$ for the blasticidin resistance gene, sat2-Fw (5'-TCGCATAGAATCGAATGGAA- $3^{\prime}$ ) and sat2-Rv ( $5^{\prime}$-GCATTCGAGTCCGTGGA- ${ }^{\prime}$ ) for the pericentromeric alphoid ${ }^{\text {chrl }}$, act-Fw (5'-GCCGGGACCTGACTGACTAC- $\left.3^{\prime}\right)$ and act-Rv (5'-AGGCTGGAAGAGTGCCTCAG-3') for actin.

\section{ASSOCIATED CONTENT}

\section{Supporting Information}

Supplementary Figures: The Supporting Information is available free of charge on the ACS Publications website at DOI: 10.1021/ acssynbio. 8 b00018.

The alphoid ${ }^{\text {hybrid }}$ HACs containing both alphoid DNA arrays, the alphoid ${ }^{\text {hybrid }}$ HAC in clone 20.CA.24 being larger than in clone 20.CA.07, structural analysis of clone HT1080-20.CA.07, and the alphoid ${ }^{\text {hybrid }}$ HAC showing epigenetically distinct centromeric domains (PDF)

\section{AUTHOR INFORMATION}

\section{Corresponding Authors}

*E-mail: omolina@carrerasresearch.org; tel: +34 93-557-2810.

*E-mail: bill.earnshaw@ed.ac.uk; tel: +44-(0)131-650-7101.

ORCID ${ }^{\circ}$

Oscar Molina: 0000-0001-7585-4519

\section{Author Contributions}

E.P.: data acquisition, analysis and interpretation, manuscript revision; N.K.: data acquisition, analysis and interpretation, wrote the manuscript, and made all arrays; N.K. and M.L.: performed Southern blot experiments; J.A.C.: contributed to the optimization of oligo-FISH experiments; V.L. and H.M.: contributed new experimental and analytical tools and revised the article critically for important intellectual content; W.C.E.: experimental design, data analysis and interpretation, manuscript drafting and revision; O.M.: experimental design, data acquisition, analysis and interpretation, wrote the manuscript.

Notes

The authors declare no competing financial interest.

\section{ACKNOWLEDGMENTS}

This work was funded by the UK Research Councils' Synthetic Biology for Growth programme core grant BB/M018040/1 to the BBSRC/EPSRC/MRC Synthetic Biological Research Centre. Additional funding of the Earnshaw lab is provided by Wellcome, of which W.C.E. is a Principal Research Fellow (grant number 073915). O.M. was funded in part by the European Molecular Biology Organization (long-term EMBO fellowship; ALTF-453-2012). Additional funding was provided by the 
Intramural Research Program of the NIH, NCI Center for Cancer Research (V.L. and N.K.) and MEXT KAKENHI (grant numbers 23247030 and 23114008) and the Kazusa DNA Research Institute Foundation (H.M.).

\section{REFERENCES}

(1) Fukagawa, T., and Earnshaw, W. C. (2014) The centromere: chromatin foundation for the kinetochore machinery,. Dev. Cell 30, 496-508.

(2) Schueler, M. G., and Sullivan, B. A. (2006) Structural and functional dynamics of human centromeric chromatin. Annu. Rev. Genomics Hum. Genet. 7, 301-313.

(3) Earnshaw, W. C., and Migeon, B. R. (1985) Three related centromere proteins are absent from the inactive centromere of a stable isodicentric chromosome. Chromosoma 92, 290-296.

(4) Sekulic, N., and Black, B. E. (2012) Molecular underpinnings of centromere identity and maintenance. Trends Biochem. Sci. 37, 220-229.

(5) Sullivan, B. A., and Schwartz, S. (1995) Identification of centromeric antigens in dicentric Robertsonian translocations: CENP$\mathrm{C}$ and CENP-E are necessary components of functional centromeres. Hum. Mol. Genet. 4, 2189-2197.

(6) du Sart, D., Cancilla, M. R., Earle, E., Mao, J. I., Saffery, R., Tainton, K. M., Kalitsis, P., Martyn, J., Barry, A. E., and Choo, K. H. (1997) A functional neo-centromere formed through activation of a latent human centromere and consisting of non-alpha-satellite DNA,. Nat. Genet. 16, 144-153.

(7) Sullivan, B. A., and Karpen, G. H. (2004) Centromeric chromatin exhibits a histone modification pattern that is distinct from both euchromatin and heterochromatin. Nat. Struct. Mol. Biol. 11, 10761083.

(8) Ribeiro, S. A., Vagnarelli, P., Dong, Y., Hori, T., McEwen, B. F., Fukagawa, T., Flors, C., and Earnshaw, W. C. (2010) A super-resolution map of the vertebrate kinetochore,. Proc. Natl. Acad. Sci. U. S. A. 107, 10484-10489.

(9) Bergmann, J. H., Rodriguez, M. G., Martins, N. M., Kimura, H., Kelly, D. A., Masumoto, H., Larionov, V., Jansen, L. E., and Earnshaw, W. C. (2011) Epigenetic engineering shows $\mathrm{H} 3 \mathrm{~K} 4 \mathrm{me} 2$ is required for HJURP targeting and CENP-A assembly on a synthetic human kinetochore. EMBO J. 30, 328-340.

(10) Harrington, J. J., Van Bokkelen, G., Mays, R. W., Gustashaw, K., and Willard, H. F. (1997) Formation of de novo centromeres and construction of first-generation human artificial microchromosomes. Nat. Genet. 15, 345-355.

(11) Ikeno, M., Grimes, B., Okazaki, T., Nakano, M., Saitoh, K., Hoshino, H., McGill, N. I., Cooke, H., and Masumoto, H. (1998) Construction of YAC-based mammalian artificial chromosomes. Nat. Biotechnol. 16, 431-439.

(12) Ebersole, T. A., Ross, A., Clark, E., McGill, N., Schindelhauer, D., Cooke, H., and Grimes, B. (2000) Mammalian artificial chromosome formation from circular alphoid input DNA does not require telomere repeats. Human molecular genetics 9, 1623-1631.

(13) Grimes, B. R., Schindelhauer, D., McGill, N. I., Ross, A., Ebersole, T. A., and Cooke, H.J. (2001) Stable gene expression from a mammalian artificial chromosome. EMBO Rep. 2, 910-914.

(14) Kouprina, N., Ebersole, T., Koriabine, M., Pak, E., Rogozin, I. B., Katoh, M., Oshimura, M., Ogi, K., Peredelchuk, M., Solomon, G., Brown, W., Barrett, J. C., and Larionov, V. (2003) Cloning of human centromeres by transformation-associated recombination in yeast and generation of functional human artificial chromosomes. Nucleic acids research 31, 922-934.

(15) Basu, J., Stromberg, G., Compitello, G., Willard, H. F., and Van Bokkelen, G. (2005) Rapid creation of BAC-based human artificial chromosome vectors by transposition with synthetic alpha-satellite arrays. Nucleic acids research 33, 587-596.

(16) Monaco, Z. L., and Moralli, D. (2006) Progress in artificial chromosome technology. Biochem. Soc. Trans. 34, 324-327.
(17) Basu, J., and Willard, H. F. (2006) Human artificial chromosomes: potential applications and clinical considerations. Pediatr. Clin. North Am. 53, 843-853.

(18) Ikeno, M., and Suzuki, N. (2011) Construction and use of a bottom-up HAC vector for transgene expression. Methods Mol. Biol. 738, $101-110$.

(19) Kouprina, N., Earnshaw, W. C., Masumoto, H., and Larionov, V. (2013) A new generation of human artificial chromosomes for functional genomics and gene therapy,. Cell. Mol. Life Sci. 70, 11351148.

(20) Kouprina, N., Tomilin, A. N., Masumoto, H., Earnshaw, W. C., and Larionov, V. (2014) Human artificial chromosome-based gene delivery vectors for biomedicine and biotechnology. Expert Opin. Drug Delivery 11, 517-535.

(21) Moralli, D., and Monaco, Z. L. (2015) Developing de novo human artificial chromosomes in embryonic stem cells using HSV-1 amplicon technology, Chromosome research: an international journal on the molecular. Chromosome Res. 23, 105-110.

(22) Oshimura, M., Uno, N., Kazuki, Y., Katoh, M., and Inoue, T. (2015) A pathway from chromosome transfer to engineering resulting in human and mouse artificial chromosomes for a variety of applications to bio-medical challenges,. Chromosome Res. 23, 111-133.

(23) Molina, O., Kouprina, N., Masumoto, H., Larionov, V., and Earnshaw, W. C. (2017) Using human artificial chromosomes to study centromere assembly and function. Chromosoma 126, 559-575.

(24) Ebersole, T., Okamoto, Y., Noskov, V. N., Kouprina, N., Kim, J. H., Leem, S. H., Barrett, J. C., Masumoto, H., and Larionov, V. (2005) Rapid generation of long synthetic tandem repeats and its application for analysis in human artificial chromosome formation. Nucleic Acids Res. 33 , e130.

(25) Kouprina, N., and Larionov, V. (2006) TAR cloning: insights into gene function, long-range haplotypes and genome structure and evolution. Nat. Rev. Genet. 7, 805-812.

(26) Kouprina, N., and Larionov, V. (2016) Transformation-associated recombination (TAR) cloning for genomics studies and synthetic biology. Chromosoma 125, 621-632.

(27) Nakano, M., Cardinale, S., Noskov, V. N., Gassmann, R. Vagnarelli, P., Kandels-Lewis, S., Larionov, V., Earnshaw, W. C., and Masumoto, H. (2008) Inactivation of a human kinetochore by specific targeting of chromatin modifiers. Dev. Cell 14, 507-522.

(28) Cardinale, S., Bergmann, J. H., Kelly, D., Nakano, M., Valdivia, M. M., Kimura, H., Masumoto, H., Larionov, V., and Earnshaw, W. C. (2009) Hierarchical inactivation of a synthetic human kinetochore by a chromatin modifier. Molecular biology of the cell 20, 4194-4204.

(29) Bergmann, J. H., Jakubsche, J. N., Martins, N. M., Kagansky, A., Nakano, M., Kimura, H., Kelly, D. A., Turner, B. M., Masumoto, H., Larionov, V., and Earnshaw, W. C. (2012) Epigenetic engineering: histone $\mathrm{H} 3 \mathrm{~K} 9$ acetylation is compatible with kinetochore structure and function. J. Cell Sci. 125, 411-421.

(30) Shono, N., Ohzeki, J., Otake, K., Martins, N. M., Nagase, T., Kimura, H., Larionov, V., Earnshaw, W. C., and Masumoto, H. (2015) CENP-C and CENP-I are key connecting factors for kinetochore and CENP-A assembly. J. Cell Sci. 128, 4572-4587.

(31) Martins, N. M., Bergmann, J. H., Shono, N., Kimura, H., Larionov, V., Masumoto, H., and Earnshaw, W. C. (2016) Epigenetic engineering shows that a human centromere resists silencing mediated by H3K27me3/K9me3. Molecular biology of the cell 27, 177-196.

(32) Ohzeki, J., Shono, N., Otake, K., Martins, N. M., Kugou, K., Kimura, H., Nagase, T., Larionov, V., Earnshaw, W. C., and Masumoto, H. (2016) KAT7/HBO1/MYST2 Regulates CENP-A Chromatin Assembly by Antagonizing Suv39h1-Mediated Centromere Inactivation. Dev. Cell 37, 413-427.

(33) Molina, O., Vargiu, G., Abad, M. A., Zhiteneva, A., Jeyaprakash, A. A., Masumoto, H., Kouprina, N., Larionov, V., and Earnshaw, W. C. (2016) Epigenetic engineering reveals a balance between histone modifications and transcription in kinetochore maintenance. Nat. Commun. 7, 13334.

(34) Carlsten, J. O., Szilagyi, Z., Liu, B., Lopez, M. D., Szaszi, E., Djupedal, I., Nystrom, T., Ekwall, K., Gustafsson, C. M., and Zhu, X. 
(2012) Mediator promotes CENP-a incorporation at fission yeast centromeres. Molecular and cellular biology 32, 4035-4043.

(35) Bourgo, R. J., Siddiqui, H., Fox, S., Solomon, D., Sansam, C. G., Yaniv, M., Muchardt, C., Metzger, D., Chambon, P., Roberts, C. W., and Knudsen, E. S. (2009) SWI/SNF deficiency results in aberrant chromatin organization, mitotic failure, and diminished proliferative capacity. Molecular biology of the cell 20, 3192-3199.

(36) Slee, R. B., Steiner, C. M., Herbert, B. S., Vance, G. H., Hickey, R J., Schwarz, T., Christan, S., Radovich, M., Schneider, B. P., Schindelhauer, D., and Grimes, B. R. (2012) Cancer-associated alteration of pericentromeric heterochromatin may contribute to chromosome instability. Oncogene 31, 3244-3253.

(37) Molina, O., Carmena, M., Maudlin, I. E., and Earnshaw, W. C. (2016) PREditOR: a synthetic biology approach to removing heterochromatin from cells, Chromosome research: an international journal on the molecular. Chromosome Res. 24, 495-509.

(38) Okamoto, Y., Nakano, M., Ohzeki, J., Larionov, V., and Masumoto, H. (2007) A minimal CENP-A core is required for nucleation and maintenance of a functional human centromere,. EMBO J. 26, 1279-1291.

(39) Masumoto, H., Ikeno, M., Nakano, M., Okazaki, T., Grimes, B., Cooke, H., and Suzuki, N. (1998) Assay of centromere function using a human artificial chromosome. Chromosoma 107, 406-416.

(40) Larin, Z., and Mejia, J. E. (2002) Advances in human artificial chromosome technology. Trends Genet. 18, 313-319.

(41) Catania, S., Pidoux, A. L., and Allshire, R. C. (2015) Sequence features and transcriptional stalling within centromere DNA promote establishment of CENP-A chromatin. PLoS Genet. 11, e1004986.

(42) Hori, T., Shang, W. H., Toyoda, A., Misu, S., Monma, N., Ikeo, K., Molina, O., Vargiu, G., Fujiyama, A., Kimura, H., Earnshaw, W. C., and Fukagawa, T. (2014) Histone H4 Lys 20 monomethylation of the CENP-A nucleosome is essential for kinetochore assembly. Dev. Cell 29, 740-749.

(43) Kouprina, N., Samoshkin, A., Erliandri, I., Nakano, M., Lee, H. S., Fu, H., Iida, Y., Aladjem, M., Oshimura, M., Masumoto, H., Earnshaw, W. C., and Larionov, V. (2012) Organization of synthetic alphoid DNA array in human artificial chromosome (HAC) with a conditional centromere. ACS Synth. Biol. 1, 590-601.

(44) Moralli, D., Jefferson, A., Valeria Volpi, E., and Larin Monaco, Z. (2013) Comparative study of artificial chromosome centromeres in human and murine cells. Eur. J. Hum. Genet. 21, 948-956.

(45) Matera, A. G., and Ward, D. C. (1992) Oligonucleotide probes for the analysis of specific repetitive DNA sequences by fluorescence in situ hybridization. Hum. Mol. Genet. 1, 535-539.

(46) Molina, O., Blanco, J., Anton, E., Vidal, F., and Volpi, E. V. (2012) High-resolution fish on DNA fibers for low-copy repeats genome architecture studies. Genomics 100, 380-386.

(47) Ohzeki, J., Bergmann, J. H., Kouprina, N., Noskov, V. N., Nakano, M., Kimura, H., Earnshaw, W. C., Larionov, V., and Masumoto, H. (2012) Breaking the HAC Barrier: histone H3K9 acetyl/methyl balance regulates CENP-A assembly. EMBO J. 31, 2391-2402.

(48) Noskov, V. N., Lee, N. C., Larionov, V., and Kouprina, N. (2011) Rapid generation of long tandem DNA repeat arrays by homologous recombination in yeast to study their function in mammalian genomes. Biol. Proced. Online 13, 8.

(49) Larionov, V., Kouprina, N., Graves, J., Chen, X. N., Korenberg, J. R., and Resnick, M. A. (1996) Specific cloning of human DNA as yeast artificial chromosomes by transformation-associated recombination. Proc. Natl. Acad. Sci. U. S. A. 93, 491-496.

(50) Kouprina, N., and Larionov, V. (2008) Selective isolation of genomic loci from complex genomes by transformation-associated recombination cloning in the yeast Saccharomyces cerevisiae. Nat. Protoc. 3, 371-377.

(51) Liskovykh, M., Lee, N. C., Larionov, V., and Kouprina, N. (2016) Moving toward a higher efficiency of microcell-mediated chromosome transfer. Mol. Ther.-Methods Clin. Dev. 3, 16043.

(52) Bodor, D. L., Mata, J. F., Sergeev, M., David, A. F., Salimian, K. J., Panchenko, T., Cleveland, D. W., Black, B. E., Shah, J. V., and Jansen, L.
E. (2014) The quantitative architecture of centromeric chromatin. eLife 3 , e02137. 